\title{
What are the Longest Ropes on the Unit Sphere?
}

\author{
Henry Gerlach \& Heiko von Der Mosel
}

\author{
Communicated by F. Отто
}

\begin{abstract}
We consider the variational problem of finding the longest closed curves of given minimal thickness on the unit sphere. After establishing the existence of solutions for any given thickness between 0 and 1 , we explicitly construct for each given thickness $\Theta_{n}:=\sin \pi /(2 n), n \in \mathbb{N}$, exactly $\varphi(n)$ solutions, where $\varphi$ is Euler's totient function from number theory. Then we prove that these solutions are unique, and also provide a complete characterisation of sphere filling curves on the unit sphere; that is of those curves whose spherical tubular neighbourhood completely covers the surface area of the unit sphere exactly once. All of these results carry over to open curves as well, as indicated in the last section.
\end{abstract}

\section{Introduction}

What is the best way to bend a bulky mattress such that most of it fits into the trunk of a car? Why is it useful to carefully roll up a long electric cable onto a cable reel in order to stow it away? This and similar questions belong to the sort of packing problems where one tries to place a maximal portion of a huge or long object into a certain volume or onto a given surface. Nature displays such optimisation tasks: it is fascinating how and in what remarkably high density extremely long strands of viral DNA are packed into the tiny volume of the phage head of a bacteriophage [16].

For the purpose of modelling complicated packing processes, it is often helpful to analyse such problems in an idealised mathematical form. For instance, one can ask: What are the longest ropes on a given surface? Specifying this target surface to be the unit sphere and realizing that ropes can be described as curves with a positive thickness, we arrive at the following packing problem: 
Problem (P). Find the longest closed curves of prescribed minimal thickness which fit onto the two-sphere $\mathbb{S}^{2}:=\left\{x \in \mathbb{R}^{3}:|x|=1\right\}$.

For a precise mathematical formulation of this maximisation problem, we recall first that the length functional $\mathscr{L}$ defined on rectifiable continuous closed curves $\gamma: \mathbb{S}^{1} \rightarrow \mathbb{R}^{3}$ is given by

$$
\mathscr{L}(\gamma):=\int_{\mathbb{S}^{1}}\left|\gamma^{\prime}(t)\right| \mathrm{d} t,
$$

where $\mathbb{S}^{1}$ denotes the unit circle $\mathbb{S}^{1} \cong \mathbb{R} / 2 \pi \mathbb{Z}$. We follow the ideas of GonZaLEZ AND MADDOCKS [12] in defining the spatial thickness $\Delta[\gamma]$ of a continuous closed curve $\gamma: \mathbb{S}^{1} \rightarrow \mathbb{R}^{3}$ of length $L>0$ with arc-length parametrisation $\Gamma:[0, L] \rightarrow$ $\mathbb{R}^{3}$ as

$$
\triangle[\gamma]:=\inf _{\substack{s \neq t \neq \tau \neq s \\ s, t, \tau \in[0, L)}} R(\Gamma(s), \Gamma(t), \Gamma(\tau))
$$

Here, $R(x, y, z)$ denotes the radius of the smallest ${ }^{1}$ circle through the points $x, y, z \in \mathbb{R}^{3}$. It was shown in [13, Lemma 2] and [8, Satz 2.14] that the arc-length parametrisation of a closed curve with positive thickness possesses a Lipschitz continuous tangent vector. This allows us to restate the variational problem $(\mathrm{P})$ more precisely:

Problem (P). Given a constant $\Theta \in(0,1]$, find a closed curve $\gamma_{\Theta}$ in the class

$$
\mathcal{C}_{\Theta}:=\left\{\gamma \in C^{1,1}\left(\mathbb{S}^{1}, \mathbb{R}^{3}\right):|\gamma|=1 \text { and }\left|\gamma^{\prime}\right|>0 \text { on } \mathbb{S}^{1}, \Delta[\gamma] \geqq \Theta\right\}
$$

such that $\mathscr{L}\left(\gamma_{\Theta}\right)=\sup _{\mathcal{C}_{\Theta}} \mathscr{L}$.

Existence problems of this kind have been solved recently and in more generality in [9]. For the convenience of the reader, however, we will present a proof of the following existence result, where we will need an extra effort to show that the minimal prescribed thickness is, in fact, attained by any solution.

Theorem 1. (Existence) For each prescribed minimal spatial thickness $\Theta \in(0,1]$, Problem (P) possesses (at least) one solution $\gamma_{\Theta} \in \mathcal{C}_{\Theta}$. In addition, every such solution has minimal thickness, that is, $\triangle\left[\gamma_{\Theta}\right]=\Theta$.

Related variational problems in the context of nonlinearly elastic curves and rods with the same notion of thickness, in particular the existence of ideal knots, were treated in [13]; for ideal knots and links see also [3,11]. Ideal knots are length minimising representatives of a given knot class subject to a prescribed minimal spatial thickness. Unfortunately, it has turned out to be quite challenging to obtain more information about the actual shape of the solutions, for example of ideal knots;

${ }^{1}$ If $x, y$, and $z$ are not collinear, $R(x, y, z)$ is simply the radius of the circumcircle of $x, y$, and $z$. If these points are collinear but distinct, we set $R(x, y, z)$ to be infinite. If they are collinear but not distinct, then $R(x, y, z)$ equals half of the diameter of the point set $\{x, y, z\}$. 


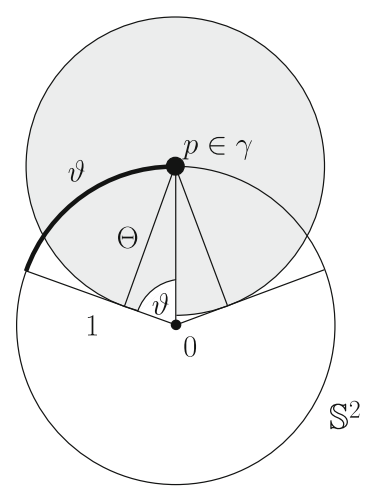

Fig. 1. The spatial tubular neighbourhood $B_{\Theta}(\gamma)$ (grey region) illuminated by a pointlike lightsource at the origin casts a shadow on the surface of the sphere, thus generating the spherical tubular neighbourhood $T_{\vartheta}(\gamma) \subset \mathbb{S}^{2}$

see $[2,7,21]$. Cantarella et al. [3, Section 3] were able to provide an explicit one-parameter family of ideal links, and in cooperation with FU AND WRINKLE [2, Section 9] analysed in detail the shape of the tight clasp, a solution of a special related boundary value problem. However, even for the simplest nontrivial knot, the trefoil, the ideal shape is analytically not determined. For numerical realizations see for example $[4,5,18,19]$.

The focus of the present paper is to construct explicit and unique solutions of (P) taking advantage of the symmetry of the target manifold $\mathbb{S}^{2}$. For the analysis on the sphere it is helpful to consider, in addition to spatial thickness, a spherical thickness. To illuminate this concept, imagine a pointlike light source being placed in the origin. For any closed curve $\gamma \subset \mathbb{S}^{2}$ the spherical tubular neighbourhood

$$
T_{\vartheta}(\gamma):=\left\{\xi \in \mathbb{S}^{2}: \operatorname{dist}_{\mathbb{S}^{2}}\left(\xi, \gamma\left(\mathbb{S}^{1}\right)\right)<\vartheta\right\}
$$

may be seen on $\mathbb{S}^{2}$ as the shadow of the spatial tubular neighbourhood $B_{\Theta}(\gamma) \subset \mathbb{R}^{3}$ for $\Theta=\sin \vartheta$; see Fig. 1. In that sense the spatial thickness $\Delta[\gamma]=\Theta$ corresponds to a spherical thickness $\vartheta=\arcsin \Theta$ (see Lemma 3 in Section 2). From now on, we will consistently use the capital letter $\Theta$ for spatial thickness values and small $\vartheta$ for spherical thickness on $\mathbb{S}^{2}$.

It has already been mentioned in [17] and [9, Remark 2.16] that for given minimal thickness $\Theta=1$, any great circle on $\mathbb{S}^{2}$ provides the unique length maximising closed curve (up to congruence). Any closed curve $\gamma$ in $\mathbb{S}^{2}$ different from a great circle has thickness $\Delta[\gamma]$ less than 1 . The great circle happens to be the first (and "simplest") member of an infinite family of solutions corresponding to the decreasing sequence of prescribed values of spatial thickness

$$
\Theta_{n}:=\sin \vartheta_{n} \quad \text { for } \vartheta_{n}:=\frac{\pi}{2 n}, n \in \mathbb{N}
$$



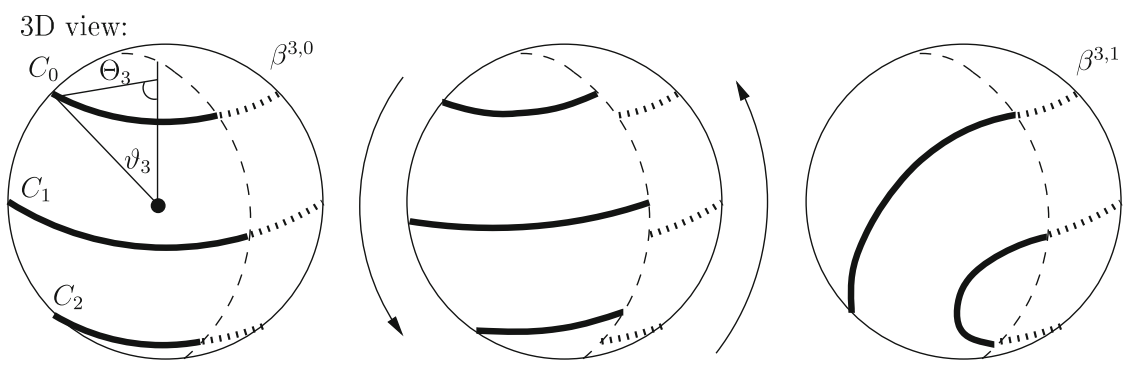

Front view:
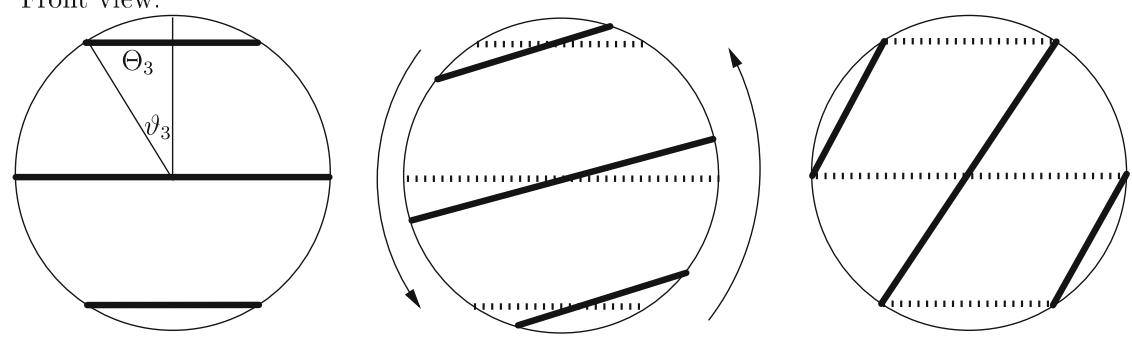

Fig. 2. Construction of $\beta^{3,1}$ starting from $\beta^{3,0}$

The building blocks of these explicit solutions will be semicircles of the $n$ latitudes

$$
C_{i}: \mathbb{S}^{1} \rightarrow \mathbb{S}^{2}, \quad i=0, \ldots, n-1
$$

Here, $C_{0}$ is a circle of spherical radius $\vartheta_{n}$ around the north pole, and all latitudinal circles $C_{i}$ have spherical distance

$$
\operatorname{dist}_{\mathbb{S}^{2}}\left(C_{i}, C_{i-1}\right)=2 \vartheta_{n} \quad \text { for } i=1, \ldots, n-1,
$$

such that the last latitude $C_{n-1}$ is a circle of spherical radius $\vartheta_{n}$ around the south pole; see Fig. 2.

We may view the union $C_{0} \cup \cdots \cup C_{n-1}$ as one (discontinuous) curve $\beta^{n, 0}$ : $\mathbb{S}^{1} \rightarrow \mathbb{S}^{2}$, where the $n$ connected components $C_{i}$ of $\beta^{n, 0}$ possess mutually disjoint tubular neighbourhoods of uniform radius $\Theta_{n}$ in $\mathbb{R}^{3}$ so that $\beta^{n, 0}$ has spatial thickness $\triangle\left[\beta^{n, 0}\right]=\Theta_{n}$. In order to construct from $\beta^{n, 0}$ continuous closed curves $\beta^{n, k}$ consisting of only one component for suitable $k \in\{1, \ldots, n-1\}$, we cut the two-sphere $\mathbb{S}^{2}$ into two hemispheres along a plane through both poles such that $\beta^{n, 0}$ is cut orthogonally into a collection of $2 n$ semicircles.

Now, we keep one hemisphere fixed while turning the other by an angle of $2 k \vartheta_{n}$ (see Fig. 2; for an animation see [10]) such that the $n$ semicircles of the original curve $\beta^{n, 0}$ on the fixed hemisphere, together with the now turned semicircles, form a closed continuous curve

$$
\beta^{n, k}: \mathbb{S}^{1} \rightarrow \mathbb{S}^{2}
$$


(a)

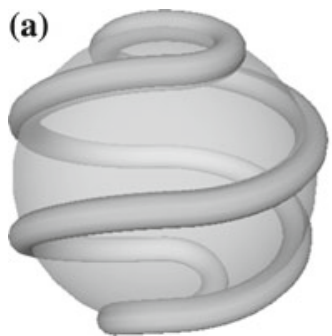

(b)

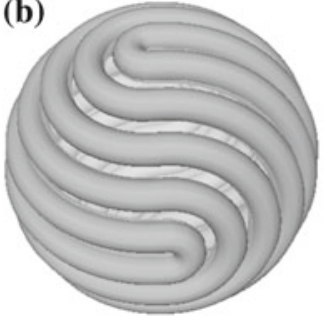

(c)

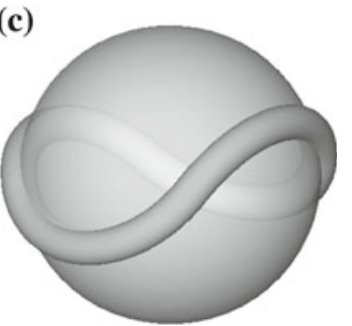

Fig. 3. a, b Solutions that maximise length for prescribed thickness. a $\beta^{4,1}$ has spatial thickness $\Theta_{4}=\sin \frac{\pi}{2 \cdot 4}$ with one hemisphere turned by $1 \cdot \Theta_{4}$. b $\beta^{12,5}$ has spatial thickness $\Theta_{12}=\sin \frac{\pi}{2 \cdot 12}$ with one hemisphere turned by $5 \cdot \Theta_{12}$. c $\beta_{\Theta}$ is a good competitor for a spatial thickness $\Theta \in\left(\Theta_{2}, \Theta_{1}\right)$. None of the curves is depicted with its full spatial thickness

That this is indeed possible under the additional algebraic condition ${ }^{2}$ that the greatest common divisor $\operatorname{gcd}(k, n)$ of $k$ and $n$ equals 1 , and that this construction leads to distinct solutions of $(\mathrm{P})$ is the content of

Theorem 2. (Explicit solutions) For each $n \in \mathbb{N}$ and $k \in\{1, \ldots, n-1\}$ with $\operatorname{gcd}(k, n)=1$ the curves $\beta^{n, k}$ are (up to rigid motions and re-parametrisations) mutually distinct members of the class $\mathcal{C}_{\Theta}$. They provide explicit piecewise circular solutions of the variational problem $(\mathrm{P})$ for prescribed minimal spatial thickness $\Theta:=\Theta_{n} \in(0,1]$. In addition, $\triangle\left[\beta^{n, k}\right]=\Theta_{n}$. For $n=1$ the equator $\beta^{1,0}$ provides the only solution with spatial thickness $\Theta_{1}=1$.

Interestingly, the solution curves $\beta^{n, k}$, some of which are depicted in Fig. 3a, b, resemble to a striking extent certain so-called Turing patterns which arise in chemistry and biology as characteristic concentration distributions of different substances as a result of a diffusion-driven instability; see for example [23].

For our variational problem $(\mathrm{P})$ we can prove that the explicit solutions for given thickness $\Theta:=\Theta_{n}, n \in \mathbb{N}$, are, in fact, unique.

Theorem 3. (Uniqueness for $\Theta=\Theta_{n}$ ) Any closed curve $\gamma_{\Theta} \in \mathcal{C}_{\Theta}$ which is a solution of $(\mathrm{P})$ for given minimal spatial thickness $\Theta=\Theta_{n}, n \in \mathbb{N}$, coincides (up to congruence and re-parametrisations) with one of the curves $\beta^{n, k}$ for $k \in\{1, \ldots, n-1\}$ with $\operatorname{gcd}(k, n)=1$. For $n=1$, that is $\Theta_{1}=1$, the equator $\beta^{1,0}$ is the unique solution. In particular, for $\Theta=\Theta_{n}$ we have exactly $\varphi(n)$ solutions for Problem $(\mathrm{P})$ where $\varphi$ denotes Euler's totient function known in number theory (see Table 1).

This uniqueness theorem is a consequence of the following stronger result which employs the two-dimensional volume $\mathscr{V}\left(T_{\vartheta}(\gamma)\right):=\mathscr{H}^{2}\left(T_{\vartheta}(\gamma)\right)$ of the tubular

2 Such a construction was used for a bead puzzle called the orb or orb it in the 1980s; see in particular the drawing on $[25$, p. 2]. The involved algebra was presumably known to its inventors. 
Table 1. The set $A_{n}:=\{k \in\{1, \ldots, n\}: \operatorname{gcd}(k, n)=1\}$ for $n \in \mathbb{N}$, consists of the values $k$, such that $\beta^{n, k}$ is a closed curve. By the uniqueness result, Theorem $3, \sharp A_{n}=\varphi(n)$ is the number of distinct solutions of Problem (P) for the thickness values $\Theta_{n}=\sin \frac{\pi}{2 n}$

\begin{tabular}{llrrrrrrrrrr}
\hline$n$ & $\sharp A_{n}$ & $n$ & $\sharp A_{n}$ & $n$ & $\sharp A_{n}$ & $n$ & $\sharp A_{n}$ & $n$ & $\sharp A_{n}$ & $n$ & $\sharp A_{n}$ \\
\hline 1 & 1 & 8 & 4 & 15 & 8 & 22 & 10 & 29 & 28 & 36 & 12 \\
2 & 1 & 9 & 6 & 16 & 8 & 23 & 22 & 30 & 8 & 37 & 36 \\
3 & 2 & 10 & 4 & 17 & 16 & 24 & 8 & 31 & 30 & 38 & 18 \\
4 & 2 & 11 & 10 & 18 & 6 & 25 & 20 & 32 & 16 & 39 & 24 \\
5 & 4 & 12 & 4 & 19 & 18 & 26 & 12 & 33 & 20 & 40 & 16 \\
6 & 2 & 13 & 12 & 20 & 8 & 27 & 18 & 34 & 16 & 41 & 40 \\
7 & 6 & 14 & 6 & 21 & 12 & 28 & 12 & 35 & 24 & 42 & 12 \\
\hline
\end{tabular}

Moreover, $\varphi(n)$ counts all sphere filling curves according to Theorem 4

neighbourhood $T_{\vartheta}(\gamma)$ on $\mathbb{S}^{2}$, to identify sphere filling curves $^{3}$ as precisely those explicit solutions $\beta^{n, k}$ for $\Theta=\Theta_{n}$ :

Theorem 4. (Sphere filling thick curves) If $\mathscr{V}\left(T_{\vartheta}(\gamma)\right)=4 \pi$ for $\vartheta \in(0, \pi / 2]$ and some closed curve $\gamma \in \mathcal{C}_{\Theta}$ with $\Theta=\sin \vartheta \in(0,1]$, then there is some $n \in \mathbb{N}$ and $k \in\{1, \ldots, n-1\}$ with $\operatorname{gcd}(k, n)=1$, or $n=1$ and $k=0$, such that (i) $\vartheta=\vartheta_{n}$, (ii) $\triangle[\gamma]=\Theta_{n}$, where $\Theta_{n}=\sin \vartheta_{n}$, and (iii) $\gamma=\beta^{n, k}$.

Notice that this theorem also provides insights about intermediate values $\Theta \in(0,1]$ of prescribed minimal thickness for arbitrary competitors $\gamma \in \mathcal{C}_{\Theta}$ :

Either $\Theta$ is one of the $\Theta_{n}$, in which case length-maximizing curves are one of the $\beta^{n, k}$, and also fill the sphere, or, for other values of $\Theta$, there are no sphere-filling curves in $C_{\Theta}$.

Our additional analysis of the relation between length, volume, and thickness reveals, among other things, an oscillatory behaviour of the volume $V(\Theta):=$ $\mathscr{V}\left(T_{\vartheta}\left(\gamma_{\Theta}\right)\right)$ as a function of the given minimal thickness $\Theta$. In Fig. 4 the black zig-zag curve serves as a lower bound for $V(\Theta)$, which is attained precisely at each $\Theta_{n}, n \in \mathbb{N}$; see Lemma 8 for the details.

Results similar to those described above are available, as well, for the problem corresponding to $(\mathrm{P})$ on open curves $\gamma:[0,1] \rightarrow \mathbb{S}^{2}$. For the details we ask the reader to consult Section 5; we refer in particular to Fig. 5 for a first impression of the shapes of length maximising open curves on the two-sphere.

Let us finally outline the structure of the paper. Section 2 contains the details of the construction of the explicit solutions $\beta^{n, k}$, where we use algebraic arguments to determine when these curves form closed single loops (Lemma 1). This section ends with the proof of Theorem 2. In Section 3 we first show how to approximate continuous loops with positive thickness by smooth loops with positive thickness, which may also be of independent interest (Lemma 4 and Corollary 1). We use this lemma twice - first to establish a variant of the well-known theorem of

${ }^{3}$ Even the equator $\beta^{1,0}$ together with its spherical tubular neighbourhood $T_{\pi / 2}\left(\beta^{1,0}\right)$ is sphere filling, although the spatial tubular neighbourhood $B_{\Theta}\left(\beta^{1,0}\right)$ covers only the considerably smaller equatorial collar $T_{\pi / 3}\left(\beta^{1,0}\right)$. 


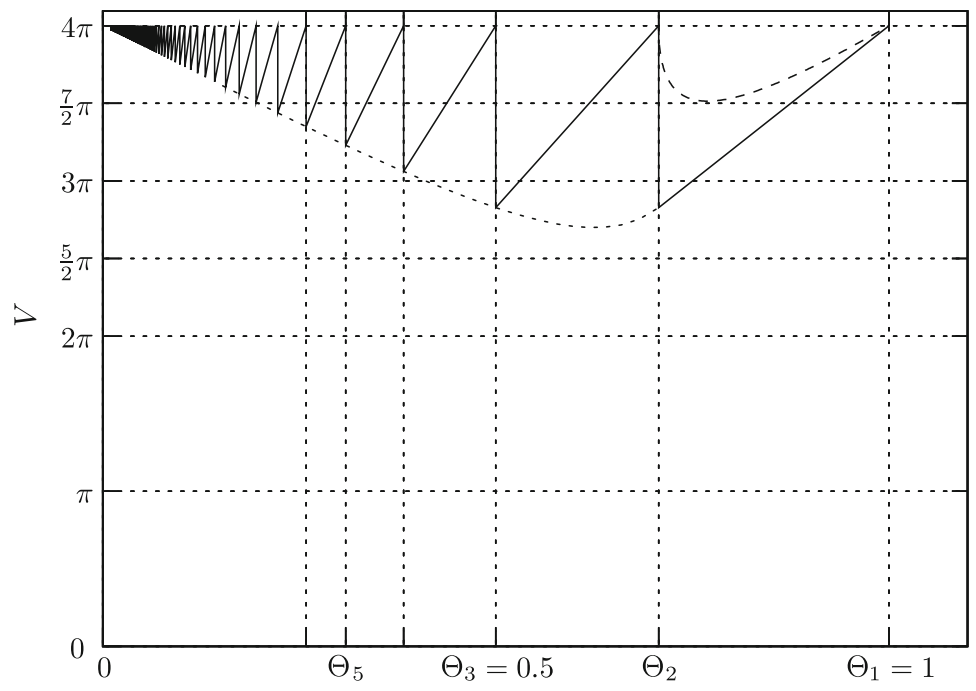

$\Theta$

Fig. 4. A lower bound for $V(\Theta)$ as established by Lemma 8. The low peaks are located at $\left(\Theta_{n+1}, 4 \pi \Theta_{n+1} / \Theta_{n}\right)$. The dotted hull-curve $h(t):=\left(\sin \left(\frac{\pi}{2 t+2}\right), 4 \pi \sin \left(\frac{\pi}{2 t+2}\right) / \sin \left(\frac{\pi}{2 t}\right)\right)$, $t \in[1, \infty)$ reveals that the spikes are not on a straight line. The values of $V\left(\Theta_{2}\right)$ and $V\left(\Theta_{3}\right)$ happen to be equal. For $\Theta \in\left(\Theta_{2}, \Theta_{1}\right)$ the volume of $\mathscr{V}\left(T_{\vartheta}\left(\beta_{\Theta}\right)\right)$ indicates (dashed line) that the curves $\beta_{\Theta}$ serve as good competitors for Problem (P), see also Fig. 8d

Hotelling and WeYL $[15,24]$ relating the volume of a non-self-intersecting tubular neighbourhood to the length of its centreline, for continuous curves with positive thickness (see Proposition 1). This is of great help in proving optimality in the variational problem $(\mathrm{P})$. The second application of the approximation result, Lemma 4, appears in the proof that the length maximiser indeed has the prescribed minimal thickness (Theorem 5). As an additional valuable tool for that purpose we construct, for any given minimal spatial thickness $\Theta \in\left(\Theta_{2}, \Theta_{1}\right)$, distinguished competing closed curves $\beta_{\Theta}$ with a construction similar to the one described above. In fact, it turns out that the two unique explicit solutions $\beta^{n, k}$ for $n=1,2$ appear as the limit members of this one-parameter family of competitors, that is, $\beta^{1,1}=\beta_{\Theta_{1}}$ and $\beta^{2,1}=\beta_{\Theta_{2}}$; see Lemma 6 and consult Fig. 4 for the corresponding tubular volumes $\mathscr{V}\left(T_{\vartheta}\left(\beta_{\Theta}\right)\right)$. At this point we are unable to prove that the curves $\beta_{\Theta}$ provide explicit solutions to (P) for the intermediate values $\Theta \in\left(\Theta_{2}, \Theta_{1}\right)$ of prescribed minimal thickness, but we view them as good candidates. Moreover, we analyse how thickness, length, and tube volume of solutions depend on the prescribed minimal thickness justifying, for example, Fig. 4; see Lemmas 7 and 8. The uniqueness result, Theorem 4 , is proved in a series of technical lemmas in Section 4. The starting point here is the observation that a spherical curve with spatial thickness $\Theta=\triangle[\gamma]$, which touches a geodesic ball of spherical radius $\vartheta:=\arcsin \Theta$ in two non-antipodal points actually contains the whole circular arc connecting these two points (Proposition 2). Then follows, with Lemma 9, a quite explicit characterisation of sphere filling curves $\gamma$ of thickness $\Theta$ : for every 
(a)

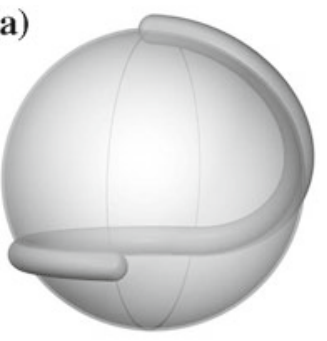

(d)

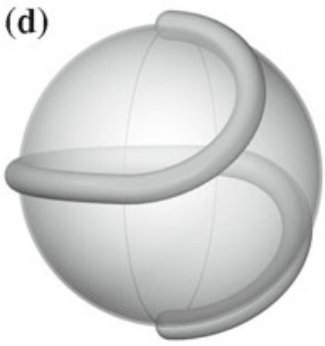

(b)

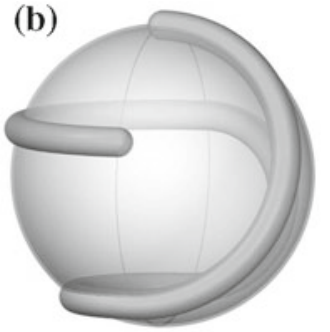

(e)

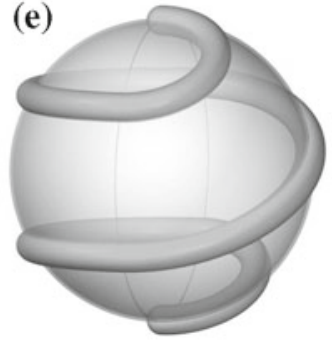

(c)

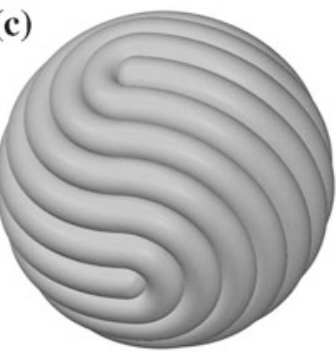

(f)

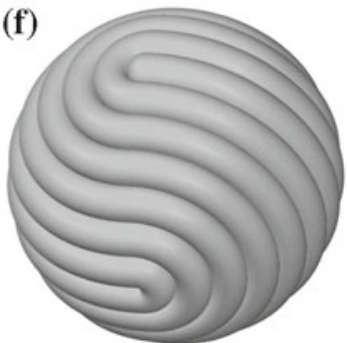

Fig. 5. Open curves $\alpha^{m, k}$ that maximise length for prescribed thicknesses $\omega_{m}=\pi / m$ : a $m=3, k=1 ; \mathbf{b} m=5, k=1$; $m=25, k=6$; d $m=4, k=0$; e $m=6, k=0$; f $m=26, k=6$. Note that the endpoints of the curve are always antipodal for $m$ even. Only the curves $\mathbf{c}$ and $\mathbf{f}$ are depicted with full thickness

open geodesic ball $\mathscr{B}_{\vartheta}$ of spherical radius $\vartheta$ that has no point in common with $\gamma$, its boundary $\partial \mathscr{B}_{\vartheta}$ intersects $\gamma$ either in a circle, a semicircle, or in two antipodal points. These two results are used in Lemmas 10 and 11 to determine characteristic patterns of sphere filling curves before we conclude with the proof of Theorem 4. In Section 5 we provide the necessary modifications to establish the analogous results for length maximising open curves on the two-sphere $\mathbb{S}^{2}$.

\section{Explicit solutions}

We recall from the introduction that we obtain the curves $\beta^{n, k}$ for $n \in \mathbb{N}$ and $k \in\{0, \ldots, n-1\}$ by cutting the two-sphere $\mathbb{S}^{2}$ into two hemispheres, from now on referred to as western hemisphere $\mathbb{S}^{w}$ and eastern hemisphere $\mathbb{S}^{e}$, such that the latitudes $C_{i}: \mathbb{S}^{1} \rightarrow \mathbb{S}^{2}$ for $i=0, \ldots, n-1$, perpendicular to the cutting plane satisfy

(i) $C_{0}$ is a circle of spherical radius $\vartheta_{n}=\pi /(2 n)$ about the north pole,

(ii) $\operatorname{dist}_{\mathbb{S}^{2}}\left(C_{i}, C_{i-1}\right)=2 \vartheta_{n}$ for $i=1, \ldots, n-1$,

which implies that $C_{n-1}$ is a circle of spherical radius $\vartheta_{n}$ about the south pole, see Fig. 2. Keeping the western hemisphere $\mathbb{S}^{w}$ fixed and turning $\mathbb{S}^{e}$ by an angle of $2 k \vartheta_{n}$ leads to a collection of $2 n$ semicircles whose (generally disconnected) union we may parametrise with constant speed to obtain our candidates $\beta^{n, k}: \mathbb{S}^{1} \rightarrow \mathbb{S}^{2}$ for $k=1, \ldots, n-1$. 
Lemma 1. For every $n \in \mathbb{N}$ and $k \in\{1, \ldots, n-1\}$ with $\operatorname{gcd}(k, n)=1$ the appropriately re-parametrised curve $\beta^{n, k}: \mathbb{S}^{1} \rightarrow \mathbb{S}^{2}$ is a closed piecewise circular curve whose constant speed parametrisation is of class $C^{1,1}\left(\mathbb{S}^{1}, \mathbb{R}^{3}\right)$ satisfying $\triangle\left[\beta^{n, k}\right]=\Theta_{n}=\sin \vartheta_{n}=\sin \frac{\pi}{2 n}$. Moreover, for distinct $k_{1}, k_{2} \in\{0, \ldots, n-1\}$, the curves $\beta^{n, k_{1}}$ and $\beta^{n, k_{2}}$ are not equivalent, that is, there is no rigid motion $M$ with $M\left(\beta^{n, k_{1}}\right)=\beta^{n, k_{2}}$.

Proof. The main issue will be to check whether the resulting curve forms a single closed embedded loop; we postpone this task and instead analyse its consequences.

Notice that by our choice of the turning angle $2 k \vartheta_{n}$ every endpoint of a semicircle on $\mathbb{S}^{w}$ meets exactly one endpoint of a semicircle on $\mathbb{S}^{e}$, so $\partial \beta^{n, k}=\varnothing$, which means that all connected components of $\beta^{n, k}$ are embedded closed loops. Moreover, our construction connects semicircles in a $C^{1}$-fashion, that is, the tangent lines of the respective semicircles coincide at the common endpoints. Once we are certain that we have obtained one single closed simple curve, we can rearrange the sub-arcs of the domain $\mathbb{S}^{1}$ corresponding to the various semicircles in the right order to obtain the desired constant speed parametrisation $\beta^{n, k}: \mathbb{S}^{1} \rightarrow \mathbb{S}^{2}$.

To show then, in addition, that $\triangle\left[\beta^{n, k}\right]=\Theta_{n}$, we recall that the original curve $\beta^{n, 0}$ consisting of the stack of $n$ disjoint latitudinal circles with mutual spherical distance $2 \vartheta_{n}$ satisfies $\triangle\left[\beta^{n, 0}\right]=\Theta_{n}$. According to [13, Lemma 3] this implies that the tubular neighbourhood $B_{\Theta_{n}}\left(\beta^{n, 0}\right)$ in $\mathbb{R}^{3}$ can be expressed as the disjoint union of normal disks, that is,

$$
B_{\Theta_{n}}\left(\beta^{n, 0}\right)=\bigcup_{s \in \mathbb{S}^{1}} D_{\Theta_{n}}\left(\beta^{n, 0}(s),\left(\beta^{n, 0}\right)^{\prime}(s)\right),
$$

where $D_{\theta}(\xi, \eta)$ denotes the two-dimensional disk of radius $\theta$ centred at $\xi \in \mathbb{R}^{3}$ and perpendicular to $\eta \in \mathbb{R}^{3} \backslash\{0\}$. Moreover, after cutting the $\mathbb{S}^{2}$ along a plane through the poles into the hemispheres $\mathbb{S}^{w}$ and $\mathbb{S}^{e}$, one observes that these normal disks centred in $\mathbb{S}^{w}$ do not intersect $\mathbb{S}^{e}$, and vice versa. Therefore also

$$
B_{\Theta_{n}}\left(\beta^{n, k}\right)=\bigcup_{s \in \mathbb{S}^{1}} D_{\Theta_{n}}\left(\beta^{n, k}(s),\left(\beta^{n, k}\right)^{\prime}(s)\right) \quad \text { for all } k=0, \ldots, n-1,
$$

since the $\beta^{n, k}$ are obtained by simply turning $\mathbb{S}^{e}$ against $\mathbb{S}^{w}$, leading to a piecewise circular closed $C^{1,1}$-curve.

We claim that for each $x \in B_{\Theta_{n}}\left(\beta^{n, k}\right)$ there is exactly one point $p \in \beta^{n, k}\left(\mathbb{S}^{1}\right)$ such that

$$
\operatorname{dist}_{\mathbb{R}^{3}}\left(x, \beta^{n, k}\right)=|x-p|,
$$

which by [13, Lemma 3 (iii) $]$ implies $\Delta\left[\beta^{n, k}\right] \geqq \Theta_{n}$, and since the local radius of curvature of the semicircles $C_{0} \cap \beta^{n, k}$ equals $\Theta_{n}$ we arrive at $\triangle\left[\beta^{n, k}\right]=\Theta_{n}$.

In order to prove the claim we use (2.1) to find a unique parameter $s=s(x) \in \mathbb{S}^{1}$ such that $x \in D_{\Theta_{n}}\left(\beta^{n, k}(s),\left(\beta^{n, k}\right)^{\prime}(s)\right)$. Since $\beta^{n, k}$ is of class $C^{1}$ we know that the segment $x-p$ is perpendicular to the curve $\beta^{n, k}$ at all points $p \in \beta^{n, k}$ satisfying (2.2). If there were one point $p:=\beta^{n, k}(t)$ satisfying (2.2) for $t \neq s$, then we would have $x \in D_{\Theta_{n}}\left(\beta^{n, k}(t),\left(\beta^{n, k}\right)^{\prime}(t)\right)$, hence

$$
x \in D_{\Theta_{n}}\left(\beta^{n, k}(s),\left(\beta^{n, k}\right)^{\prime}(s)\right) \cap D_{\Theta_{n}}\left(\beta^{n, k}(t),\left(\beta^{n, k}\right)^{\prime}(t)\right) \neq \emptyset
$$




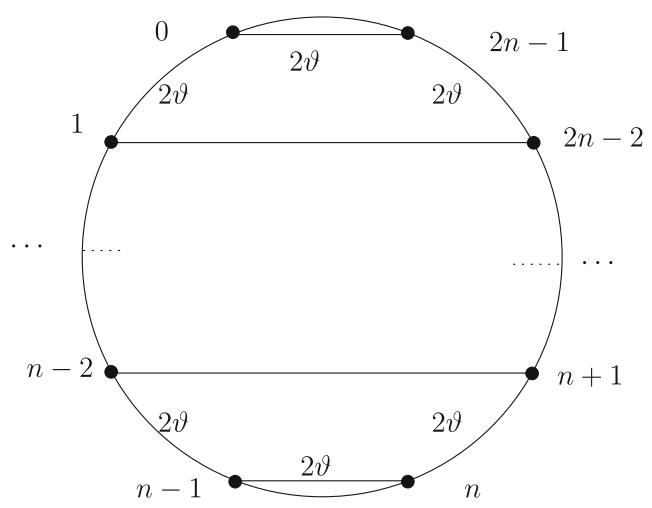

Fig. 6. Labelling $2 n$ checkpoints on a hemisphere for Lemma 1

contradicting the fact that the sets on the right-hand side of (2.1) are disjoint. So the only point satisfying (2.2) is the point $p:=\beta^{n, k}(s)$, which proves the claim.

It remains to be shown that each of the $\beta^{n, k}$ with $\operatorname{gcd}(k, n)=1$ forms one single closed loop. For that purpose we introduce certain checkpoints and study if and how the curve $\beta^{n, k}$ passes through these points. We consider the fixed western hemisphere $\mathbb{S}^{w}$ and label the $2 n$ endpoints of the semicircles counter-clockwise from 0 to $2 n-1$, such that checkpoints number $i$ and $2 n-1-i$ correspond to the $i$ th semicircle on $\mathbb{S}^{w}$ for $i=0, \ldots, n-1$; see Fig. 6 . The $n$ semicircles on $\mathbb{S}^{w}$ connect the checkpoints to $n$ pairs which may be viewed as a permutation $c:=\left(\begin{array}{ll}0 & 2 n-1\end{array}\right)\left(\begin{array}{ll}1 & 2 n-2\end{array}\right) \cdots(n-1 \quad n)$ consisting of $n$ cycles of length 2 , or alternatively,

$$
c(i) \equiv-1-i \quad \bmod 2 n, \quad i=0, \ldots, 2 n-1 .
$$

So if we pass through checkpoint $i$ along the corresponding semicircle contained in $\beta^{n, k} \cap \mathbb{S}^{w}$, we will next pass through checkpoint $c(i)$ as the endpoint of this semicircle upon entering the eastern hemisphere $\mathbb{S}^{e}$. To model the turn of the other hemisphere $\mathbb{S}^{e}$ by an angle of $2 \vartheta_{n}$ against $\mathbb{S}^{w}$ we use the permutation $t:=\left(\begin{array}{lllll}0 & 1 & \cdots & 2 n-2 & 2 n-1\end{array}\right)$ consisting of 1 cycle of length $2 n$, so that the turning angle of $2 k \vartheta_{n}$ corresponds to

$$
t^{k}(i) \equiv i+k \quad \bmod 2 n \text { for } k=1, \ldots, n-1 .
$$

As we proceed along the curve $\beta^{n, k}$, we pass alternately through the semicircles on $\mathbb{S}^{w}$ and the semicircles on the rotated hemisphere $\mathbb{S}^{e}$, respectively. It can easily be checked that if we enter $\mathbb{S}^{w}$ through checkpoint $i$ then we enter $\mathbb{S}^{w}$ the next time at checkpoint

$$
q(i):=t^{-k} \circ c \circ t^{k} \circ c(i) \quad \bmod 2 n, \quad i=0, \ldots, 2 n-1 .
$$

We enter through $n$ distinct checkpoints and therefore pass through all semicircles on $\mathbb{S}^{w}$ and $\mathbb{S}^{e}$, respectively, if and only if the permutation $q=t^{-k} \circ c \circ t^{k} \circ c$ consists 
of exactly two cycles of common length $n$. In order to determine under which conditions this happens, we calculate $q(i) \equiv i-2 k \bmod 2 n$, hence $q^{l}(i) \equiv i-2 k l$ mod $2 n$. This relation shows, in particular, that the reentry after an even checkpoint is again an even checkpoint, ensuring that no semicircle on $\mathbb{S}^{w}$ and, for symmetry reasons also on $\mathbb{S}^{e}$, is left out in the process.

By Lemma 2, which will also be applied in the construction of open curve solutions in Section 5, we conclude that $q$ consists of two cycles of common length $n$ if and only if $(2 n) / \operatorname{gcd}(2 k, 2 n)=n \Leftrightarrow \operatorname{gcd}(k, n)=1$, otherwise it consists of $2 \operatorname{gcd}(k, n)$ cycles of length $n / \operatorname{gcd}(k, n)$. To see the latter we note that the cyclic group $\langle q\rangle:=\left\{q, q^{2}, q^{3}, \ldots\right\}$ operates freely on the set of checkpoints. The number of algebraically disjoint orbits, that is cycles in our situation, is given by the well-known orbit formula

$$
\sharp \text { orbits of }\langle q\rangle\}=\frac{\sharp \text { checkpoints }\}}{|\langle q\rangle|}=\frac{2 n}{n / \operatorname{gcd}(k, n)}=2 \operatorname{gcd}(k, n) .
$$

Switching the roles of the entry and exit checkpoints on the western hemisphere, $S^{w}$, will produce the opposite orientation of the constructed curves. Since each algebraic orbit corresponds to one of the two opposite orientations, we end up with $\operatorname{gcd}(k, n)$ closed loops, in particular with one closed curve if $\operatorname{gcd}(k, n)=1$.

To see that for $k_{1} \neq k_{2}$ the curve $\beta^{n, k_{1}}$ cannot be mapped by a rigid motion to $\beta^{n, k_{2}}$, we consider the oriented angle between the polar axes connecting the respective north and south poles on $\mathbb{S}^{w}$ and the tilted $\mathbb{S}^{e}$ measured counterclockwise in the cutting plane as seen from $\mathbb{S}^{w}$. This invariant under rigid motions is, in fact, different for $k_{1} \neq k_{2}$. This finishes the proof of Lemma 1 .

Lemma 2. For given $r, v \in \mathbb{N}$ let the permutation $q:\{0, \ldots, r-1\} \longrightarrow$ $\{0, \ldots, r-1\}$ be defined as $q(i):=i-v \bmod r$. Then the orbit length of $i$ under the cyclic group $\langle q\rangle$ is $\sharp\left\{q^{0}(i), \ldots, q^{r}(i)\right\}=r / \operatorname{gcd}(v, r)$.

Proof. For $l=r / \operatorname{gcd}(v, r)$ we find

$q^{l}(i) \equiv i-v \frac{r}{\operatorname{gcd}(v, r)} \equiv i-r \frac{v}{\operatorname{gcd}(v, r)} \equiv i \quad \bmod r \quad$ for all $i=0, \ldots, r-1$,

which proves that no cycle in $q$ is longer than $r / \operatorname{gcd}(v, r)$. Now let $m>0$ be the smallest integer such that

$$
q^{m}(i) \equiv i-v m \equiv i \quad \bmod r
$$

for some $i \in\{0, \ldots, r-1\}$. By (2.3) vm is a multiple of $r$, that is there exists $j \in \mathbb{N}$ such that

$$
v m=j \operatorname{lcm}(v, r)=j v r / \operatorname{gcd}(v, r),
$$

where $\operatorname{lcm}(v, r)$ denotes the least common multiple of $v$ and $r$. Cancelling $v$ yields $m=j r / \operatorname{gcd}(v, r)$, which implies $m \geqq r / \operatorname{gcd}(v, r)$. But $r / \operatorname{gcd}(v, r)$ is also an upper bound for $m$ as shown above and consequently $m=r / \operatorname{gcd}(v, r)$. 


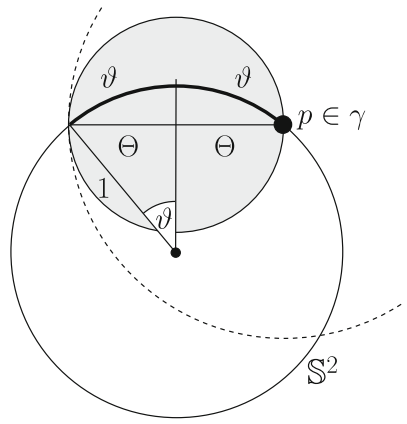

Fig. 7. The unit sphere cut along a normal plane that is orthogonal to $\gamma$ at the point $p \in \gamma$. The grey spatial ball rotated along the dashed circle generates a geodesic ball $\mathscr{B}_{\vartheta} \subset \mathbb{S}^{2}$ of radius $\vartheta=\arcsin \Theta$ such that the boundary $\partial \mathscr{B}_{\vartheta}$ is tangent to $\gamma$ and $\mathscr{B}_{\vartheta} \cap \gamma\left(\mathbb{S}^{1}\right)=\emptyset$

Remark 1. (i) Since we are only interested in different shapes, we do not have to take into account any integer $k \geqq n$, since the corresponding $\operatorname{arcs} \beta^{n, k} \cap \mathbb{S}^{e}$ are equivalent with $\beta^{n, k-n} \cap \mathbb{S}^{e}$ for $k \geqq n .^{4}$ Therefore, the number of distinct closed curves $\beta^{n, k}$ for given $n \in \mathbb{N}$ is identical with the cardinality of the set

$$
A_{n}:=\{k \in\{1, \ldots, n\}: \operatorname{gcd}(k, n)=1\}, \quad n \in \mathbb{N},
$$

which equals $\varphi(n)$, where $\varphi$ is Euler's totient function (see for example [1, p. 21]). In Table 1 we have listed the number of distinct closed curves of type $\beta^{n, k}$ for $n \leqq 42$.

(ii) If we turn the hemisphere $\mathbb{S}^{e}$ in Lemma 1 by the angle $k \cdot 2 \vartheta_{n}$ for some $k$ with $\operatorname{gcd}(k, n)>1$ the curve splits into $\operatorname{gcd}(k, n)$ connected components. This configuration is a solution to an optimisation problem similar to $(\mathrm{P})$, namely maximising the length of collections of precisely $\operatorname{gcd}(n, k)$ closed curves on $\mathbb{S}^{2}$ subject to the prescribed minimal thickness $\Theta_{n}$.

In order to show that the constructed curves $\beta^{n, k}$ actually maximise length, we are going to use a version of Hotelling's famous theorem relating tube volume to the length of the tube's centreline. For this, we recall from [13, Lemma 3 (i)] the torus property (T): Let $\Delta[\gamma] \geqq \Theta>0$. Then the union of all open balls $B_{\Theta}$ of radius $\Theta$ which are tangent to the curve $\gamma$ at any fixed point $p \in \gamma$ has no point in common with $\gamma$. This readily implies (see Fig. 7) the

Spherical torus property (ST): Any closed spherical curve $\gamma: \mathbb{S}^{1} \rightarrow \mathbb{S}^{2}$ with spatial thickness $\triangle[\gamma] \geqq \Theta$ satisfies

$$
\gamma\left(\mathbb{S}^{1}\right) \cap \mathscr{B}_{\vartheta}(\xi)=\varnothing \quad \text { for } \vartheta=\arcsin \Theta
$$

for any geodesic open ball

$$
\mathscr{B}_{\vartheta}(\xi):=\left\{\eta \in \mathbb{S}^{2}: \operatorname{dist}_{\mathbb{S}^{2}}(\eta, \xi)<\vartheta\right\}
$$

whose boundary $\partial \mathscr{B}_{\vartheta}(\xi)$ is tangent to $\gamma$ in at least one point of $\gamma$.

\footnotetext{
${ }^{4}$ For algebraic reasons we do count the case $k=n$ instead of $k=0$.
} 
Definition 1. (Tubes without self-overlap) Let $\gamma: \mathbb{S}^{1} \rightarrow \mathbb{S}^{2}$ be a spherical closed curve which possesses a tangent at every point. The spherical tubular neighbourhood

$$
T_{\phi}(\gamma):=\left\{x \in \mathbb{S}^{2}: \operatorname{dist}_{\mathbb{S}^{2}}(x, \gamma)<\phi\right\}
$$

is said to be non-self-overlapping if two geodesic arcs of length $\phi$ emanating from two distinct curve points in a direction perpendicular to $\gamma$ have, at most, common endpoints, but otherwise do not intersect. In that case we say that $\gamma$ has spherical thickness at least $\phi$.

Lemma 3. (Thick curves and non-self-overlapping tubes) All spherical tubular neighbourhoods $T_{\phi}(\gamma)=\left\{x \in \mathbb{S}^{2}: \operatorname{dist}_{\mathbb{S}^{2}}(x, \gamma)<\phi\right\}, \phi \in(0, \vartheta]$, of a spherical closed curve $\gamma: \mathbb{S}^{1} \rightarrow \mathbb{S}^{2}$ with spatial thickness $\triangle[\gamma] \geqq \Theta=\sin \vartheta$ for $\vartheta \in(0, \pi / 2]$ are non-self-overlapping.

Proof. Recall, first, from [13, Lemma 2] that $\gamma$ possesses a $C^{1,1}$-arclength parametrization so that $\gamma$ indeed possesses a tangent vector at every curve point. It suffices to prove the statement for $\phi=\vartheta$. Now, assume, to the contrary, that two geodesic arcs of length $\vartheta$ emanating perpendicularly from $\gamma$ from two different curve points $p$ and $q$ have one point $x \in \mathbb{S}^{2}$ in common which is not the endpoint of both arcs. We can assume without loss of generality that $\operatorname{dist}_{\mathbb{S}^{2}}(x, q) \leqq \operatorname{dist}_{\mathbb{S}^{2}}(x, p)$. Let $p^{\prime}$ be the endpoint of the geodesic ray emanating from $p$ and $q^{\prime}$ the endpoint of the geodesic ray emanating from $q$. Then, by the triangle inequality,

$$
\operatorname{dist}_{\mathbb{S}^{2}}\left(p^{\prime}, q\right)<\operatorname{dist}_{\mathbb{S}^{2}}\left(p^{\prime}, x\right)+\operatorname{dist}_{\mathbb{S}^{2}}(x, q) \leqq \operatorname{dist}_{\mathbb{S}^{2}}\left(q^{\prime}, x\right)+\operatorname{dist}_{\mathbb{S}^{2}}(x, q)=\vartheta,
$$

where the strict inequality holds unless $p^{\prime}$ lies on the geodesic arc connecting $q$ and $q^{\prime}$, in which case $p^{\prime} \neq q^{\prime}$ since $p \neq q$. In both cases, however, we find that the geodesic ball $\mathscr{B}_{\vartheta}\left(p^{\prime}\right)$ whose boundary $\partial \mathscr{B}_{\vartheta}\left(p^{\prime}\right)$ is tangent to $\gamma$ in $p$ contains the point $q \in \gamma$, contradicting the spherical torus property (ST).

Proof of Theorem 2. According to Lemma 1 and Remark 1 (i) we find for each $n \in \mathbb{N}$ exactly $\varphi(n)$ distinct closed curves $\beta^{n, k} \in \mathcal{C}_{\Theta_{n}}$ with $\triangle\left[\beta^{n, k}\right]=\Theta_{n}$. By construction, the spherical tubular neighbourhood $T_{\vartheta_{n}}\left(\beta^{n, k}\right)$ covers the two-sphere except for a set of two-dimensional measure zero:

$$
\mathscr{V}\left(T_{\vartheta_{n}}\left(\beta^{n, k}\right)\right)=\mathscr{H}^{2}\left(T_{\vartheta_{n}}\left(\beta^{n, k}\right)\right)=4 \pi=\mathscr{H}^{2}\left(\mathbb{S}^{2}\right) .
$$

Moreover, $T_{\vartheta_{n}}\left(\beta^{n, k}\right)$ is non-self-overlapping in the sense of Definition 1. Hence by virtue of the well-known theorem of HotelLing [15] (see also [14,24]), which we are going to adapt to the present context of thick loops in Proposition 1, one has

$$
\mathscr{L}(\gamma)=\frac{\mathscr{V}\left(T_{\vartheta_{n}}\left(\beta^{n, k}\right)\right)}{2 \sin \vartheta_{n}} \leqq \frac{4 \pi}{2 \Theta_{n}}=\frac{2 \pi}{\Theta_{n}} \quad \text { for all } \gamma \in \mathcal{C}_{\Theta_{n}} .
$$

This estimate produces a sharp uniform upper bound on the length functional on the class $\mathcal{C}_{\Theta_{n}}$. Regarding (2.4), this bound is attained by the curves $\beta^{n, k}$, which means that they are length maximising in the class $\mathcal{C}_{\Theta_{n}}$, that is, their smooth and regular parametrisations are solutions of Problem $(\mathrm{P})$. 


\section{Existence, and properties of thickness, length and volume, of general solutions}

For the volume $\mathscr{V}\left(T_{\vartheta}(\gamma)\right)$ of the spherical tube $T_{\vartheta}(\gamma)=\left\{x \in \mathbb{S}^{2}: \operatorname{dist}_{\mathbb{S}^{2}}(x, \gamma)\right.$ $<\vartheta$ \} on the sphere $\mathbb{S}^{2}$, we will prove the following version of the theorem of HoTELLING [15] for continuous thick curves:

Proposition 1. Let $\gamma: \mathbb{S}^{1} \rightarrow \mathbb{S}^{2}$ be a closed rectifiable continuous curve with thickness $\Delta[\gamma]>0$ and length $\mathscr{L}(\gamma)$. Then for all $\vartheta \in[0, \arcsin (\Delta[\gamma])]$ one has

$$
\mathscr{V}\left(T_{\vartheta}(\gamma)\right)=2 \mathscr{L}(\gamma) \sin \vartheta
$$

In particular, the spherical tubular neighbourhood $T_{\arcsin \Theta}\left(\gamma_{\Theta}\right)$ of any solution $\gamma_{\Theta} \in \mathcal{C}_{\Theta}$ of Problem $(\mathrm{P})$ for given thickness $\Theta \in(0,1]$ covers the same amount of area on $\mathbb{S}^{2}$.

The obvious idea to prove this result is to approximate such thick curves by smooth ones with controlled minimal thickness for which the classic result of Hotelling is applicable and then go to the limit. That this is, indeed, possible is guaranteed by the following lemma, which we will also use for a variational argument later on in this section to show that length maximisers attain the prescribed minimal thickness; see Theorem 5 .

Lemma 4. (Smooth approximation with positive thickness I) Let $\gamma \in C^{1}\left(\mathbb{S}^{1}, \mathbb{R}^{3}\right)$ be a closed and regular curve with positive thickness $\Delta[\gamma]>0$. Then for any sequence $\left\{\gamma_{j}\right\} \subset C^{1,1}\left(\mathbb{S}^{1}, \mathbb{R}^{3}\right)$ satisfying

(i) $\gamma_{j} \rightarrow \gamma$ in $C^{1}\left(\mathbb{S}^{1}, \mathbb{R}^{3}\right)$ as $j \rightarrow \infty$,

(ii) $\lim \sup _{j \rightarrow \infty}\left\|\kappa_{j}\right\|_{L^{\infty}((0,2 \pi))} \leqq \frac{1}{\Delta[\gamma]}$, where $\kappa_{j}$ denotes the local curvature ${ }^{5}$ of $\gamma_{j}$ for $j \in \mathbb{N}$,

one has

$$
\liminf _{j \rightarrow \infty} \Delta\left[\gamma_{j}\right] \geqq \triangle[\gamma] .
$$

Proof. The length $L:=\mathscr{L}(\gamma)$ is positive since $\Delta[\gamma]>0$, and by [13, Lemma 2] the arc-length parametrisation $\Gamma:[0, L] \rightarrow \mathbb{R}^{3}$ is of class $C^{1,1}\left([0, L], \mathbb{R}^{3}\right)$. Notice furthermore that the $C^{1}$-convergence we assume in (i) implies

$$
L_{j}:=\mathscr{L}\left(\gamma_{j}\right) \rightarrow \mathscr{L}(\gamma)=L \quad \text { and } \quad\left|\gamma_{j}^{\prime}\right| \rightarrow\left|\gamma^{\prime}\right|>0 \quad \text { on } \quad \mathbb{S}^{1} \quad \text { as } j \rightarrow \infty \text {. }
$$

The arc-length parametrisations $\Gamma_{j}:\left[0, L_{j}\right] \rightarrow \mathbb{R}^{3}$ satisfy by Assumption (ii)

$$
\limsup _{j \rightarrow \infty}\left\|\Gamma_{j}^{\prime \prime}\right\|_{L^{\infty}\left(\left(0, L_{j}\right), \mathbb{R}^{3}\right)}=\limsup _{j \rightarrow \infty}\left\|\kappa_{j}\right\|_{L^{\infty}((0,2 \pi))} \leqq \frac{1}{\triangle[\gamma]} .
$$

5 By Assumption (i) we can assume that all $\gamma_{j}$ are regular curves, and recall that $C^{1,1}\left(\mathbb{S}^{1}, \mathbb{R}^{3}\right) \cong W^{2, \infty}\left((0,2 \pi), \mathbb{R}^{3}\right)$ so that $\kappa_{j}$ exists and is bounded almost everywhere on $(0,2 \pi)$ for each $j \in \mathbb{N}$. 
In order to establish (3.2) it suffices to show that for any given $\epsilon>0$ there is some $j_{0}=j_{0}(\epsilon) \in \mathbb{N}$ such that

$$
\triangle\left[\gamma_{j}\right]=\triangle\left[\Gamma_{j}\right] \geqq(1-\epsilon) \Delta[\gamma] \quad \text { for all } j \geqq j_{0} .
$$

We argue by contradiction, so if we assume on the contrary that there exists an $\epsilon_{0}>0$ such that $\Delta\left[\Gamma_{j}\right]<\left(1-\epsilon_{0}\right) \triangle[\gamma]$ for a subsequence $j \rightarrow \infty$, then for each member $j$ of this subsequence we can find an arc-length parameter $s_{j} \in\left[0, L_{j}\right]$ such that by definition of thickness [see (1.1)]

$$
\rho_{G}\left[\gamma_{j}\right]\left(s_{j}\right)<\left(1-\frac{\epsilon_{0}}{2}\right) \triangle[\gamma],
$$

where $\rho_{G}\left[\gamma_{j}\right]$ denotes the global radius of curvature of $\gamma_{j}$ defined in $[12,13]$ by

$$
\rho_{G}\left[\gamma_{j}\right]\left(s_{j}\right):=\inf _{\substack{t, \tau \in\left[0, L_{j}\right] \backslash\left\{s_{j}\right\} \\ t \neq \tau}} R\left(\Gamma_{j}\left(s_{j}\right), \Gamma_{j}(t), \Gamma_{j}(\tau)\right) .
$$

It was shown in [22, Lemma 5] that

$$
\rho_{G}\left[\gamma_{j}\right]\left(s_{j}\right)=\rho_{\mathrm{pt}}\left[\gamma_{j}\right]\left(s_{j}\right):=\inf _{\tau \in\left[0, L_{j}\right] \backslash\left\{s_{j}\right\}} \operatorname{pt}\left[\gamma_{j}\right]\left(s_{j}, \tau\right),
$$

where $\mathrm{pt}\left[\gamma_{j}\right]\left(s_{j}, \tau\right)$ denotes the radius of the unique circle through the points $\Gamma_{j}\left(s_{j}\right)$ and $\Gamma_{j}(\tau)$ which is tangent to the curve $\Gamma_{j}$ at the point $\Gamma_{j}(\tau)$. Therefore, we can find for each $j$ some arc-length parameter $\sigma_{j} \in\left[0, L_{j}\right] \backslash\left\{s_{j}\right\}$ such that by (3.5)

$$
\operatorname{pt}\left[\gamma_{j}\right]\left(s_{j}, \sigma_{j}\right)<\left(1-\frac{\epsilon_{0}}{4}\right) \triangle[\gamma]
$$

Going back to the original parametrisation $\gamma_{j}: \mathbb{S}^{1} \rightarrow \mathbb{R}^{3}$, we find parameters $t_{j}, \tau_{j} \in \mathbb{S}^{1}$ given by

$$
\int_{0}^{t_{j}}\left|\gamma_{j}^{\prime}(z)\right| \mathrm{d} z=s_{j} \neq \sigma_{j}=\int_{0}^{\tau_{j}}\left|\gamma_{j}^{\prime}(z)\right| \mathrm{d} z,
$$

and by choice of an appropriate subsequence we may assume that $\left(t_{j}, \tau_{j}\right) \rightarrow$ $(t, \tau) \in \mathbb{S}^{1} \times \mathbb{S}^{1}$ as $j \rightarrow \infty$. Two cases may occur: either those limit parameters $t$ and $\tau$ are distinct or they coincide.

Case I. If $t \neq \tau$ then $\gamma(t) \neq \gamma(\tau)$ since $\gamma$ is simple, and therefore also $\Gamma(s) \neq$ $\Gamma(\sigma)$ for $s:=\int_{0}^{t}\left|\gamma^{\prime}(z)\right| \mathrm{d} z$ and $\sigma:=\int_{0}^{\tau}\left|\gamma^{\prime}(z)\right| \mathrm{d} z$. (Notice that we assumed that $\gamma$ is a regular curve, that is, $\left|\gamma^{\prime}\right|>0$ so that a double point $\gamma(t)=\gamma(\tau)$ for $t \neq \tau$ would imply a double point for the arc-length parametrisation $\Gamma(s)=\Gamma(\sigma)$, which is impossible because $\Delta[\gamma]$ is positive.) We arrive at

$$
\Gamma_{j}\left(s_{j}\right)=\gamma\left(t_{j}\right) \rightarrow \gamma(t)=\Gamma(s) \quad \text { and } \quad \Gamma_{j}\left(\sigma_{j}\right)=\gamma\left(\tau_{j}\right) \rightarrow \gamma(\tau)=\Gamma(\sigma)
$$

as $j \rightarrow \infty$. In addition, one has for the derivatives by the $C^{1}$-convergence, and in particular by (3.3),

$$
\Gamma_{j}^{\prime}\left(\sigma_{j}\right)=\frac{\gamma_{j}^{\prime}\left(\tau_{j}\right)}{\left|\gamma_{j}^{\prime}\left(\tau_{j}\right)\right|} \longrightarrow \frac{\gamma^{\prime}(\tau)}{\left|\gamma^{\prime}(\tau)\right|}=\Gamma^{\prime}(\sigma) \quad \text { as } j \rightarrow \infty,
$$


so that we can use an explicit formula for the pt-radius and [22, Lemma 4] to obtain from (3.6)

$$
\begin{aligned}
\left(1-\frac{\epsilon_{0}}{4}\right) \Delta[\gamma] & \underset{(3.6)}{\longrightarrow} \operatorname{pt}\left[\gamma_{j}\right]\left(s_{j}, \sigma_{j}\right)=\frac{\left|\Gamma_{j}\left(s_{j}\right)-\Gamma_{j}\left(\sigma_{j}\right)\right|^{2}}{2\left|\left(\Gamma_{j}\left(s_{j}\right)-\Gamma_{j}\left(\sigma_{j}\right)\right) \wedge \Gamma_{j}^{\prime}\left(\sigma_{j}\right)\right|} \\
& \stackrel{\mid j \rightarrow \infty}{\longrightarrow} \frac{|\Gamma(s)-\Gamma(\sigma)|^{2}}{2\left|(\Gamma(s)-\Gamma(\sigma)) \wedge \Gamma^{\prime}(\sigma)\right|}=\operatorname{pt}[\gamma](s, \sigma) \\
& \geqq \rho_{\mathrm{pt}}[\gamma](s) \underset{[22, \mathrm{~L} .4]}{\geqq} \rho_{G}[\gamma](s) \geqq \Delta[\gamma],
\end{aligned}
$$

which is a contradiction.

Case II. If $t=\tau$ we find $\left|t_{j}-\tau_{j}\right| \rightarrow 0$ as $j \rightarrow \infty$, so that by (3.3) for $j \gg 1$

$$
\left|s_{j}-\sigma_{j}\right|=\left|\int_{\tau_{j}}^{t_{j}}\right| \gamma_{j}^{\prime}(z)|\mathrm{d} z| \leqq 2\left\|\gamma^{\prime}\right\|_{C^{0}\left(\mathbb{S}^{1}, \mathbb{R}^{3}\right)}\left|t_{j}-\tau_{j}\right| \rightarrow 0 \quad \text { as } j \rightarrow \infty .
$$

We apply (3.4) to the Taylor expansion (see [22, (2.20)])

$$
\operatorname{pt}\left[\gamma_{j}\right]\left(s_{j}, \sigma_{j}\right)=\frac{\left|\Gamma_{j}^{\prime}\left(\sigma_{j}\right)+f_{\left[\sigma_{j}, s_{j}\right]} \int_{\sigma_{j}}^{u} \Gamma_{j}^{\prime \prime}(z) \mathrm{d} z d u\right|^{2}}{2\left|\Gamma_{j}^{\prime}\left(\sigma_{j}\right) \wedge \frac{1}{\sigma_{j}-s_{j}} \int_{0}^{1} \int_{\sigma_{j}-u\left(\sigma_{j}-s_{j}\right)}^{\sigma_{j}} \Gamma_{j}^{\prime \prime}(z) \mathrm{d} z d u\right|}
$$

to find for given $\delta>0$ some $j_{1}=j_{1}(\delta)$ such that for all $j \geqq j_{1}$

$\left|\int_{\sigma_{j}}^{u} \Gamma_{j}^{\prime \prime}(z) \mathrm{d} z\right| \leqq \int_{\left[\sigma_{j}, s_{j}\right]}\left|\Gamma_{j}^{\prime \prime}(z)\right| \mathrm{d} z \underset{(3.4)}{\leqq}(1+\delta)\left|s_{j}-\sigma_{j}\right| \frac{1}{\triangle[\gamma]} \quad$ for all $u \in\left[\sigma_{j}, s_{j}\right]$,

and

$$
\left|\int_{\sigma_{j}-u\left(\sigma_{j}-s_{j}\right)}^{\sigma_{j}} \Gamma_{j}^{\prime \prime}(z) \mathrm{d} z\right| \underset{(3.4)}{\leqq}(1+\delta)|u|\left|s_{j}-\sigma_{j}\right| \frac{1}{\triangle[\gamma]} \quad \text { for all } u \in[0,1] .
$$

This together with (3.8) and (3.9) allows us to estimate the numerator in (3.10) by $1-\delta$ from below, and the denominator by $(1+\delta) / \triangle[\gamma]$ from above for all $j \geqq j_{2}$ for some $j_{2}=j_{2}(\delta) \geqq j_{1}$. We infer from (3.6)

$$
\left(1-\frac{\epsilon_{0}}{4}\right) \Delta[\gamma]>\operatorname{pt}\left[\gamma_{j}\right]\left(s_{j}, \sigma_{j}\right) \geqq \frac{1-\delta}{1+\delta} \Delta[\gamma] \quad \text { for all } j \geqq j_{2},
$$

which is absurd for any $\delta \leqq \epsilon_{0} /\left(8-\epsilon_{0}\right)$.

Now we present the

Proof of Proposition 1. The length $L:=\mathscr{L}(\gamma)$ is positive since $\Delta[\gamma]>0$. Recall from [13, Lemma 2] that the arc-length parametrisation $\Gamma:[0, L] \rightarrow \mathbb{S}^{2}$ is injective and of class $C^{1,1}\left([0, L], \mathbb{R}^{3}\right)$ satisfying the local curvature bound

$$
\left\|\Gamma^{\prime \prime}\right\|_{L^{\infty}\left([0, L], \mathbb{R}^{3}\right)} \leqq \frac{1}{\triangle[\gamma]} .
$$


We extend the components $\Gamma^{i}, i=1,2,3$, as $L$-periodic functions onto all of $\mathbb{R}$. Then we choose a sequence $\epsilon_{j} \rightarrow 0$ as $j \rightarrow \infty$, a standard nonnegative mollifier $\phi \in C_{0}^{\infty}((-1,1))$, and define the smooth $L$-periodic convolutions

$$
\eta_{j}^{i}:=\phi_{\epsilon_{j}} * \Gamma^{i} \in C^{\infty}(\mathbb{R}) \subset C^{\infty}([0, L]) \text { for } i=1,2,3,
$$

so that $\eta_{j}:=\left(\eta_{j}^{1}, \eta_{j}^{2}, \eta_{j}^{3}\right)$ are smooth closed curves in $\mathbb{R}^{3}$ approximating $\Gamma$ in $C^{1}\left([0, L], \mathbb{R}^{3}\right)$, such that

$$
\begin{aligned}
& \left|\eta_{j}^{\prime}\right| \longrightarrow 1 \text { uniformly on }[0, L], \quad \text { and also } \\
& \left\|\eta_{j}^{\prime \prime}\right\|_{L^{\infty}\left([0, L], \mathbb{R}^{3}\right)} \leqq\left\|\Gamma^{\prime \prime}\right\|_{L^{\infty}\left([0, L], \mathbb{R}^{3}\right)} \leqq \frac{1}{\triangle[\gamma]} \quad \text { for all } j \in \mathbb{N} .
\end{aligned}
$$

Furthermore, we can assume that $\left|\eta_{j}\right|>0$ for all $j$, such that the projected curves

$$
\gamma_{j}:=\frac{\eta_{j}}{\left|\eta_{j}\right|}:[0, L] \rightarrow \mathbb{S}^{2}
$$

are well-defined and of class $C^{\infty}\left([0, L], \mathbb{R}^{3}\right)$ for all $j \in \mathbb{N}$. One can easily check that

$$
\gamma_{j} \longrightarrow \Gamma \quad \text { in } C^{1}\left([0, L], \mathbb{R}^{3}\right) \quad \text { as } j \rightarrow \infty \text {. }
$$

The normal curvature of $\Gamma:[0, L] \rightarrow \mathbb{S}^{2}$ equals -1 almost everywhere on $[0, L]$ (see for example [6, Chapter 3.2]) so that in particular $\Gamma^{\prime \prime} \cdot \Gamma=-1$ almost everywhere on $[0, L]$. This, together with (3.11), can be used to estimate

$$
\left|\eta_{j}(s) \cdot \eta_{j}^{\prime \prime}(s)-\Gamma(s) \cdot \Gamma^{\prime \prime}(s)\right| \leqq 2\|\Gamma(s-\cdot)-\Gamma(s)\|_{C^{0}\left(\overline{B_{\epsilon_{j}}(0)}, \mathbb{R}^{3}\right)} \frac{1}{\triangle[\gamma]},
$$

so that

$$
\left\|\eta_{j} \cdot \eta_{j}^{\prime \prime}-\Gamma \cdot \Gamma^{\prime \prime}\right\|_{L^{\infty}((0, L))} \longrightarrow 0 \quad \text { as } j \rightarrow \infty .
$$

In addition, since $\eta_{j} \rightarrow \Gamma$ in $C^{1}\left([0, L], \mathbb{R}^{3}\right)$ and $\Gamma \cdot \Gamma^{\prime} \equiv 0$ we find

$$
-\frac{\eta_{j} \cdot \eta_{j}^{\prime}}{\left|\eta_{j}\right|^{3}} \eta_{j}^{\prime}-\frac{3\left(\eta_{j} \cdot \eta_{j}^{\prime}\right)^{2}}{\left|\eta_{j}\right|^{5}} \eta_{j}-\frac{\eta_{j} \cdot \eta_{j}^{\prime}}{\left|\eta_{j}\right|^{3}} \eta_{j}^{\prime} \longrightarrow 0 \text { in } C^{0}\left([0, L], \mathbb{R}^{3}\right) \text { as } j \rightarrow \infty
$$

which, together with (3.12), implies

$$
\limsup _{j \rightarrow \infty}\left\|\gamma_{j}^{\prime \prime}\right\|_{L^{\infty}\left((0, L), \mathbb{R}^{3}\right)}=\limsup _{j \rightarrow \infty}\left\|\eta_{j}^{\prime \prime}\right\|_{L^{\infty}\left((0, L), \mathbb{R}^{3}\right)} \leqq \frac{1}{\Delta[\gamma]} .
$$

This, in combination with (3.13), implies for the arc-length parametrisations $\Gamma_{j}:\left[0, \mathscr{L}\left(\gamma_{j}\right)\right] \rightarrow \mathbb{S}^{2}$ the estimate

$$
\limsup _{j \rightarrow \infty}\left\|\Gamma_{j}^{\prime \prime}\right\|_{L^{\infty}\left((0, L), \mathbb{R}^{3}\right)}=\limsup _{j \rightarrow \infty}\left\|\frac{\left|\gamma_{j}^{\prime \prime} \wedge \gamma_{j}^{\prime}\right|}{\left|\gamma_{j}^{\prime}\right|^{3}}\right\|_{L^{\infty}\left((0, L), \mathbb{R}^{3}\right)} \leqq \frac{1}{\triangle[\gamma]} .
$$


With this inequality and with (3.13) we have verified Assumptions (i) and (ii) of Lemma 4 for the curve $\Gamma \in C^{1,1}\left([0, L], \mathbb{R}^{3}\right)$ and the approximating curves $\gamma_{j}:[0, L] \rightarrow \mathbb{S}^{2}$ each of class $C^{\infty}\left([0, L], \mathbb{R}^{3}\right)$, so that by (3.2)

$$
\liminf _{j \rightarrow \infty} \Delta\left[\gamma_{j}\right] \geqq \Delta[\Gamma]=\Delta[\gamma] .
$$

If we we take an appropriate subsequence and relabel, we obtain a sequence $\left\{\gamma_{j}\right\} \subset C^{\infty}\left([0, L], \mathbb{R}^{3}\right), \gamma_{j}:[0, L] \rightarrow \mathbb{S}^{2}$ for all $j \in \mathbb{N}$ such that

$$
\left\|\gamma_{j}-\Gamma\right\|_{C^{1}\left([0, L], \mathbb{R}^{3}\right)}<\frac{1}{j} \quad \text { and } \quad \Delta\left[\gamma_{j}\right]>\Delta[\gamma]-\frac{1}{j} .
$$

In particular, we find for arbitrary given $\vartheta \in(0, \arcsin (\triangle[\gamma])]$ and $\epsilon>0$ some $J_{0}=J_{0}(\epsilon)$ such that

$$
T_{\vartheta-\epsilon-(2 / j)}\left(\gamma_{j}\right) \subset T_{\vartheta-\epsilon-(1 / j)}(\gamma) \subset T_{\vartheta-\epsilon}\left(\gamma_{j}\right) \text { for all } j \geqq J_{0},
$$

and according to Lemma 3 these nested spherical tubular neighbourhoods are nonself-overlapping in the sense of Definition 1. Since the $\gamma_{j}$ are smooth with image on $\mathbb{S}^{2}$ for all $j$, we may apply the theorem of HoteLLING [15] twice for $j \geqq J_{1}$ for some $J_{1}=J_{1}(\epsilon) \geqq J_{0}$ with

$$
\Delta\left[\gamma_{j}\right] \underset{(3.16)}{>} \Delta[\gamma]-\frac{1}{j} \geqq \sin (\vartheta-\epsilon),
$$

to obtain for all $j \geqq J_{1}$

$$
\begin{aligned}
2 \sin (\vartheta-\epsilon-(2 / j)) \mathscr{L}\left(\gamma_{j}\right) & \underset{[15]}{=} \mathscr{V}\left(T_{\vartheta-\epsilon-(2 / j)}\left(\gamma_{j}\right)\right) \underset{(3.17)}{\leqq} \mathscr{V}\left(T_{\vartheta-\epsilon-(1 / j)}(\gamma)\right) \\
& \leqq \mathscr{V}\left(T_{\vartheta-\epsilon}\left(\gamma_{j}\right)\right) \underset{[15]}{=} 2 \sin (\vartheta-\epsilon) \mathscr{L}\left(\gamma_{j}\right) .
\end{aligned}
$$

Since (3.16) implies the convergence $\mathscr{L}\left(\gamma_{j}\right) \rightarrow \mathscr{L}(\gamma)$ as $j \rightarrow \infty$, we arrive at $2 \sin (\vartheta-\epsilon) \mathscr{L}(\gamma)=\mathscr{V}\left(T_{\vartheta-\epsilon}(\gamma)\right)$ for the arbitrarily chosen $\epsilon>0$, which implies (3.1).

We actually established within the previous proof the following approximation result, which might also be of independent interest.

Corollary 1. (Smooth approximation with positive thickness II). For any closed, continuous and rectifiable curve $\gamma: \mathbb{S}^{1} \rightarrow \mathbb{R}^{3}$ with positive thickness $\Delta[\gamma]>$ 0 and length $L:=\mathscr{L}(\gamma)$, there is a sequence of regular closed curves $\eta_{j} \in C^{\infty}\left([0, L], \mathbb{R}^{3}\right)$ such that

$$
\eta_{j} \rightarrow \Gamma \quad \text { in } C^{1} \quad \text { as } j \rightarrow \infty \quad \text { and } \quad \liminf _{j \rightarrow \infty} \Delta\left[\eta_{j}\right] \geqq \Delta[\gamma] \text {, }
$$

where $\Gamma:[0, L] \rightarrow \mathbb{R}^{3}$ denotes the arc-length parametrisation of $\gamma$. If, in addition, $\gamma\left(\mathbb{S}^{1}\right) \subset \mathbb{S}^{2}$, then there is a sequence of regular closed curves $\gamma_{j} \in C^{\infty}\left([0, L], \mathbb{R}^{3}\right)$ with $\gamma_{j}([0, L]) \subset \mathbb{S}^{2}$ such that (3.18) holds for $\gamma_{j}$ instead of $\eta_{j}$. 
As a prerequisite for the proof of the existence result, Theorem 1, we show that the velocities of constant speed parametrisations of admissible curves $\gamma \in \mathcal{C}_{\Theta}$ are controlled solely in terms of the given thickness $\Theta$ :

Lemma 5. (Speed limit) For all $\gamma \in \mathcal{C}_{\Theta}$ with $\left|\gamma^{\prime}\right| \equiv$ const. on $\mathbb{S}^{1}$ one has

$$
\Theta \leqq\left|\gamma^{\prime}\right| \leqq \frac{1}{\Theta} \quad \text { on } \mathbb{S}^{1}
$$

Proof. The upper bound in (3.19) follows from (3.1) in Proposition 1 for $\vartheta=\arcsin \Theta$ :

$$
2 \pi\left|\gamma^{\prime}\right|=\mathscr{L}(\gamma) \underset{(3.1)}{=} \frac{1}{2 \sin \vartheta} \mathscr{V}\left(T_{\vartheta}(\gamma)\right) \leqq \frac{4 \pi}{2 \Theta},
$$

and the lower bound follows from the torus property (T) (see Remark 1), which implies that $\gamma$, as a closed curve of positive length, has to be at least as long as a great circle on one of the spheres $\partial B_{\Theta}$ touching $\gamma$ in, say $\gamma(0)$, so that $2 \pi\left|\gamma^{\prime}\right|=$ $\mathscr{L}(\gamma) \geqq 2 \pi \Theta$.

Proof of Theorem 1 for closed curves. ${ }^{6}$ The class $\mathcal{C}_{\Theta}$ is not empty for any $\Theta \in(0,1]$, since any great circle $c_{g}$ smoothly parametrised with constant speed has thickness $\triangle\left[c_{g}\right]=1 \geqq \Theta$. So there is a maximising sequence $\left\{\eta_{j}\right\} \subset \mathcal{C}_{\Theta}$ such that $L_{j}:=\mathscr{L}\left(\eta_{j}\right) \rightarrow \sup _{\mathcal{C}_{\Theta}} \mathscr{L}(\cdot)$ as $j \rightarrow \infty$. The corresponding arc-length parametrisations $\Gamma_{j}:\left[0, L_{j}\right] \rightarrow \mathbb{S}^{2}$ satisfy the uniform estimate (see [13, Lemma 2])

$$
\begin{aligned}
\left\|\Gamma_{j}\right\|_{C^{1,1}\left(\left[0, L_{j}\right], \mathbb{R}^{3}\right)} & =\left\|\Gamma_{j}\right\|_{C^{0}\left(\left[0, L_{j}\right], \mathbb{R}^{3}\right)}+\left\|\Gamma_{j}^{\prime}\right\|_{C^{0}\left(\left[0, L_{j}\right], \mathbb{R}^{3}\right)}+\left\|\Gamma_{j}^{\prime \prime}\right\|_{L^{\infty}\left(\left(0, L_{j}\right), \mathbb{R}^{3}\right)} \\
& \leqq 2+\frac{1}{\Theta} \quad \text { for all } j \in \mathbb{N}
\end{aligned}
$$

so that the constant speed re-parametrisations $\gamma_{j}: \mathbb{S}^{1} \rightarrow \mathbb{S}^{2}$ with $v_{j}:=\left|\gamma_{j}^{\prime}\right|>0$ still yield a maximising sequence in $\mathcal{C}_{\Theta}$ and satisfy $\gamma_{j}(t)=\Gamma_{j}\left(t v_{j}\right)$ and $\gamma_{j}^{\prime}(t)=$ $\Gamma_{j}^{\prime}\left(t v_{j}\right) v_{j}$. Therefore by (3.19) in Lemma 5

$$
\begin{aligned}
\left|\gamma_{j}^{\prime}(t)-\gamma_{j}^{\prime}(\tau)\right| & =v_{j}\left|\Gamma_{j}^{\prime}\left(t v_{j}\right)-\Gamma_{j}^{\prime}\left(\tau v_{j}\right)\right| \\
& \leqq \frac{v_{j}^{2}}{\Theta}|t-\tau| \leqq \frac{1}{\Theta^{3}}|t-\tau| \quad \text { for all } t, \tau \in \mathbb{S}^{1} .
\end{aligned}
$$

Consequently, we obtain the uniform bound

$$
\begin{aligned}
\left\|\gamma_{j}\right\|_{C^{1,1}\left(\mathbb{S}^{1}, \mathbb{R}^{3}\right)} & \leqq\left\|\gamma_{j}\right\|_{C^{0}\left(\mathbb{S}^{1}, \mathbb{R}^{3}\right)}+v_{j}\left\|\Gamma_{j}^{\prime}\right\|_{C^{0}\left(\left[0, L_{j}\right], \mathbb{R}^{3}\right)}+\frac{1}{\Theta^{3}} \\
& \leqq 1+\frac{1}{\Theta}+\frac{1}{\Theta^{3}} \quad \text { for all } j \in \mathbb{N}
\end{aligned}
$$

which implies by the theorem of Arzelà-Ascoli the existence of a closed curve $\gamma_{\Theta} \in C^{1,1}\left(\mathbb{S}^{1}, \mathbb{R}^{3}\right)$ and a subsequence $\left\{\gamma_{j}\right\}$ such that $\gamma_{j} \longrightarrow \gamma_{\Theta}$ in $C^{1}\left(\mathbb{S}^{1}, \mathbb{R}^{3}\right)$ as

\footnotetext{
${ }^{6}$ For open curves we will indicate the necessary modifications in Section 5.
} 
$j \rightarrow \infty$. Hence $\gamma_{\Theta}$ maps $\mathbb{S}^{1}$ into $\mathbb{S}^{2}$ and has constant speed $\left|\gamma_{\Theta}^{\prime}\right| \geqq \Theta$ because $^{7}$ $\left|\gamma_{j}^{\prime}\right| \geqq \Theta$ for all $j$ by Lemma 5. In addition, $\Delta\left[\gamma_{\Theta}\right] \geqq \Theta$, since it was proved in $[20$, Lemma 4] and [11, Lemma 5] that the thickness $\Delta[\cdot]$ is upper semi-continuous with respect to convergence in $C^{0}\left(\mathbb{S}^{1}, \mathbb{R}^{3}\right)$ subject to a uniform upper bound on length. Thus we have shown that $\gamma_{\Theta} \in \mathcal{C}_{\Theta}$. Since the length functional $\mathscr{L}(\cdot)$ is continuous with respect to $C^{1}$-convergence we conclude $\sup _{\mathcal{C}_{\Theta}} \mathscr{L}(\cdot) \geqq \mathscr{L}\left(\gamma_{\Theta}\right)=$ $\lim _{j \rightarrow \infty} \mathscr{L}\left(\gamma_{j}\right)=\sup _{\mathcal{C}_{\Theta}} \mathscr{L}(\cdot)$, that is, $\gamma_{\Theta}$ is a length maximising curve. That the prescribed thickness is attained by any solution $\gamma_{\Theta}$ of Problem (P), that is $\Delta\left[\gamma_{\Theta}\right]=\Theta$, is the content of the next theorem, which then concludes the proof of Theorem 1.

Theorem 5. (Thickness is attained) For any solution $\gamma_{\Theta} \in \mathcal{C}_{\Theta}$ of Problem (P) for $\Theta \in(0,1]$ one has $\triangle\left[\gamma_{\Theta}\right]=\Theta$.

Proof. There is nothing to prove for $\Theta=1$ since then the great circle with thickness 1 is the unique solution (up to congruence). Assuming $\Delta\left[\gamma_{\Theta}\right]>\Theta$ for some $\Theta \in(0,1)$ we will first use Lemma 4 to show that variations of the type $\left(\gamma_{\Theta}+\right.$ $\epsilon \psi) /\left|\gamma_{\Theta}+\epsilon \psi\right|$ are admissible for $\epsilon$ sufficiently small, to conclude with a variational argument that $\gamma_{\Theta}$ must be a great circle. Then we construct a suitable comparison curve $\beta_{\Theta *}$ with $\mathscr{L}\left(\beta_{\Theta *}\right)>\mathscr{L}\left(\gamma_{\Theta}\right)$, which contradicts the maximality of $\gamma_{\Theta}$.

For brevity, we set $\gamma:=\gamma_{\Theta}$ for fixed $\Theta \in(0,1)$ and $L:=\mathscr{L}(\gamma)$, and we may assume, without loss of generality, that $\left|\gamma^{\prime}\right| \equiv$ const. $=: v$ on $\mathbb{S}^{1}$; hence $v=L /(2 \pi)$.

We claim that for any $\psi \in C_{0}^{\infty}\left(\mathbb{S}^{1}, \mathbb{R}^{3}\right)$ there is some $\epsilon_{0}=\epsilon_{0}(\psi, \Delta[\gamma])>0$ such that for the curves $\eta_{\epsilon}:=(\gamma+\epsilon \psi) /|\gamma+\epsilon \psi| \in C^{1,1}\left(\mathbb{S}^{1}, \mathbb{R}^{3}\right)$ we have $\triangle\left[\eta_{\epsilon}\right]>\Theta$ for all $\epsilon \in\left[-\epsilon_{0}, \epsilon_{0}\right]$.

For the proof of this claim we notice that $|\gamma|=1$, so that $\gamma \cdot \gamma^{\prime}=0$ on $\mathbb{S}^{1}$, which implies $\eta_{\epsilon} \rightarrow \gamma$ in $C^{0}\left(\mathbb{S}^{1}, \mathbb{R}^{3}\right)$ as $\epsilon \rightarrow 0$, and

$\eta_{\epsilon}^{\prime}=\frac{\gamma^{\prime}+\epsilon \psi^{\prime}}{|\gamma+\epsilon \psi|}-\frac{(\gamma+\epsilon \psi) \cdot\left(\gamma^{\prime}+\epsilon \psi^{\prime}\right)}{|\gamma+\epsilon \psi|^{3}}(\gamma+\epsilon \psi) \rightarrow \gamma^{\prime} \quad$ in $C^{0}\left(\mathbb{S}^{1}, \mathbb{R}^{3}\right) \quad$ as $\epsilon \rightarrow 0$.

As in the proof of Proposition 1 we use the fact that the arc-length parametrisation $\Gamma$ of the spherical curve $\gamma$ satisfies $\Gamma \cdot \Gamma^{\prime \prime}=-1$ almost everywhere on $[0, L]$, so that we obtain with $\Gamma^{\prime}(s)=\frac{\gamma^{\prime}}{\left|\gamma^{\prime}\right|}(t(s))=\frac{\gamma^{\prime}(t(s))}{v}$ and $\Gamma^{\prime \prime}=\frac{\gamma^{\prime \prime}(t(s))}{v} \frac{d t}{d s}(s)=$ $\frac{\gamma^{\prime \prime}(t(s))}{v^{2}}$ for the arc-length parameters $s:=\int_{0}^{t(s)}\left|\gamma^{\prime}(\tau)\right| \mathrm{d} \tau=v t(s)$ the relation $-1=\Gamma \cdot \Gamma^{\prime \prime}=\frac{\gamma \cdot \gamma^{\prime \prime}}{v^{2}}$, or

$$
\gamma \cdot \gamma^{\prime \prime}=-v^{2} \quad \text { almost everywhere on } \mathbb{S}^{1} \text {. }
$$

7 It is also possible to prove $\left|\gamma_{\Theta}^{\prime}\right| \geqq 1$ by comparing the length of the maximiser $\gamma_{\Theta}$ to that of a great circle which has thickness 1 and is henceforth an admissible comparison curve in $\mathcal{C}_{\Theta}$ for any $\Theta \in(0,1]$. 
This, together with $\gamma \cdot \gamma^{\prime}=0$ and the boundedness of $\|\gamma\|_{C^{1,1}\left(\mathbb{S}^{1}, \mathbb{R}^{3}\right)}$, can be used in a direct calculation to deduce for the curvature $\kappa_{\epsilon}$ of $\eta_{\epsilon}$

$$
\begin{aligned}
\limsup _{\epsilon \rightarrow 0}\left\|\kappa_{\epsilon}\right\|_{L^{\infty}((0,2 \pi))} & =\limsup _{\epsilon \rightarrow 0}\left\|\frac{\left|\eta_{\epsilon}^{\prime \prime} \wedge \eta_{\epsilon}^{\prime}\right|}{\left|\eta_{\epsilon}^{\prime}\right|^{3}}\right\|_{L^{\infty}((0,2 \pi))} \\
& \leqq\left\|\Gamma^{\prime \prime}\right\|_{L^{\infty}\left((0,2 \pi), \mathbb{R}^{3}\right)} \leqq \frac{1}{\Delta[\gamma]}
\end{aligned}
$$

Lemma 4 applied to $\gamma=\gamma_{\Theta}$ and $\gamma_{j}:=\eta_{\epsilon_{j}}$ for any subsequence $\epsilon_{j} \rightarrow 0$ gives $\liminf _{j \rightarrow \infty} \Delta\left[\eta_{\epsilon_{j}}\right] \geqq \Delta[\gamma]>\Theta$, so that we, indeed, find $\epsilon_{0}=\epsilon_{0}(\psi, \Delta[\gamma])>0$ such that $\Delta\left[\eta_{\epsilon}\right]>\Theta$ for all $\epsilon \in\left[-\epsilon_{0}, \epsilon_{0}\right]$, which proves the claim.

Therefore, we have $\eta_{\epsilon} \in \mathcal{C}_{\Theta}$ for all $\epsilon \in\left[-\epsilon_{0}, \epsilon_{0}\right]$ and

$$
\mathscr{L}(\gamma) \geqq \mathscr{L}\left(\eta_{\epsilon}\right) \quad \text { for all } \epsilon \in\left[-\epsilon_{0}, \epsilon_{0}\right] .
$$

Since $|\gamma+\epsilon \psi|>0$ for all $|\epsilon| \ll 1$ and $|\gamma|=1$ on $\mathbb{S}^{1}$, we can calculate the vanishing first variation of $\mathscr{L}$ at $\gamma$ :

$$
0=\frac{d}{d \epsilon}\left[\mathscr{L}\left(\eta_{\epsilon}\right)\right]_{\epsilon=0}=v^{-1} \int_{0}^{2 \pi}\left[\gamma^{\prime} \cdot \psi^{\prime}-v^{2}(\gamma \cdot \psi)\right] \mathrm{d} t .
$$

Integrating by parts and applying the Fundamental Lemma in the calculus of variations, we obtain $\gamma^{\prime \prime}+v^{2} \gamma=0$ almost everywhere on $\mathbb{S}^{1}$. Since $\gamma \in C^{1,1}$, we obtain immediately $\gamma^{\prime \prime} \in C^{1,1}$ and by the standard bootstrap argument, finally, $\gamma \in C^{\infty}\left(\mathbb{S}^{1}, \mathbb{R}^{3}\right)$. Transforming the equation into the arc-length formulation we obtain $\Gamma^{\prime \prime}=-\Gamma$ on $[0, L]$, which have great circles as their only solutions; see for example [6, p. 246]. Hence, we have shown that if $\Delta[\gamma]>\Theta$ for the solution $\gamma=\gamma_{\Theta}$, then $\gamma_{\Theta}$ is a great circle.

For each $\tau \in\left[\Theta_{2}, 1\right)$ we will construct in Lemma 6, below, a competitor $\beta_{\tau} \in \mathcal{C}_{\tau}$ with $\mathscr{L}\left(\beta_{\tau}\right)>2 \pi=\mathscr{L}\left(\gamma_{\Theta}\right)$ so that we obtain for the special choice $\tau=\Theta^{*}:=\max \left\{\Theta_{2}, \Theta\right\}$ a competitor $\beta_{\tau} \in \mathcal{C}_{\Theta^{*}} \subset \mathcal{C}_{\Theta}$, which leads to the desired contradiction against the maximality of $\mathscr{L}\left(\gamma_{\Theta}\right)$ in $\mathcal{C}_{\Theta}$.

Lemma 6. (Explicit competitors $\beta_{\tau}$ for $\tau \in\left[\Theta_{2}, \Theta_{1}\right]$ ) For every $\tau \in\left[\Theta_{2}, \Theta_{1}\right]=$ $[\sin (\pi / 4), 1]$ there is a closed curve $\beta_{\tau} \in \mathcal{C}_{\tau}$ with $\triangle\left[\beta_{\tau}\right]=\tau$,

$$
\mathscr{L}\left(\beta_{\tau}\right)=8 \tau \arccos \sqrt{1-\frac{1}{2 \tau^{2}}}, \quad \text { and } \quad \mathscr{V}\left(\beta_{\tau}\right)=2 \tau \mathscr{L}\left(\beta_{\tau}\right) .
$$

In particular, $\mathscr{L}\left(\beta_{\tau}\right) \geqq 2 \pi$ with equality if and only if $\beta_{\tau}$ is a great circle, that is if $\tau=\Theta_{1}=1$.

Remark. Our construction will reveal a one-parameter family $\left\{\beta_{\tau}\right\}$ (parametrised by the prescribed thickness $\tau$ ) continuously joining the unique solutions for $\tau=\Theta_{1}=1$ and $\tau=\Theta_{2}$ (see Fig. 4).

We strongly believe that these $\beta_{\tau}$ provide the unique (but not sphere-filling) solutions for every $\tau \in\left(\Theta_{2}, \Theta_{1}\right)$, which would extend our uniqueness result, Theorem 3 , to this continuous range of given thickness values. Up to now, however, we have no proof for this conjecture. We only would like to point out at this moment that the curves $\beta_{\tau}$ are good candidates for the maximisers for $\tau \in\left(\Theta_{2}, \Theta_{1}\right)$. 
Proof of Lemma 6. To construct $\beta_{\tau}$ for given $\tau \in\left[\Theta_{2}, \Theta_{1}\right]$ we equipartition the equator of the $\mathbb{S}^{2}$ by four distinct points $P_{i}, i=1,2,3,4$, such that these four points are the vertices of a square of edge length $2 H:=2 \cdot \frac{1}{\sqrt{2}}$ in the plane $E$ containing the equator. Now we take the plane vertical to $E$ which contains the straight segment $P_{1} P_{2}$ and rotate that plane about the rotations axis through $P_{1}$ and $P_{2}$ until this rotated plane $F$ intersected with $\mathbb{S}^{2}$ is a circle $\partial B_{\tau}$ of radius $\tau$; see Fig. 8a. Let $\alpha=\alpha(\tau)$ be the angle between $F$ and $E$, and we refer to the side-view in Fig. 8 b where $E$ is seen as a horizontal line, to obtain the geometric identities $a=H \cos \alpha, h=H \sin \alpha, \tau=\sqrt{1-h^{2}}=\sqrt{1-H^{2} \sin ^{2} \alpha}$. In Fig. 8c, the plane $F$ coincides with the drawing plane, and one can read off the relation $L=2 \tau \beta=2 \tau \arccos (a / \tau)$, where $L$ denotes the length of the shorter circular arc on $\partial B_{\tau}$ with endpoints $P_{1}$ and $P_{2}$. Repeating this process for the other edges $P_{2} P_{3}$, $P_{3} P_{4}$ and $P_{4} P_{1}$, we obtain four such circular arcs, each of length $L$. Reflecting two of these arcs opposite across the equatorial plane $E$ and taking the union of these reflections with the two remaining arcs, we obtain the desired $C^{1,1}$-curve $\beta_{\tau}$, which by construction ${ }^{8}$ has length $4 L=8 \tau \arccos (a / \tau)=8 \tau \arccos \sqrt{1-\frac{1}{2 \tau^{2}}}$. The thickness of $\beta_{\tau}$ is realized exclusively by the local radius of curvature $\tau$ of each circular arc, since neighbouring arcs are separated by the plane $S$ containing the normal disk of radius $\tau$ at the common endpoint. All normal disks of radius $\tau$ centred on one of these arcs are not only mutually disjoint but also completely contained in the half-space bounded by $S$ that contains the arc itself. (Compare with our argument to prove (2.1) in Section 2 in the construction of the explicit solutions $\beta^{n, k}$, whose circular arcs were full semicircles, whereas the arcs to build $\beta_{\tau}$ for $\tau \in\left(\Theta_{2}, \Theta_{1}\right)$ are strict subsets of semicircles.) And the normal disks of opposite arcs can also not intersect if $\sqrt{1-H^{2} \sin ^{2} \alpha}=\tau \leqq d:=\frac{H}{\sin \alpha}$; see Fig. 8b, which is true since the function $f(\alpha):=\frac{1}{2}-\sin ^{2} \alpha+\frac{1}{2} \sin ^{4} \alpha$ satisfies $f(\pi / 2)=0$ and is monotonically decreasing on $[0, \pi / 2]$.

Thus we have shown $\triangle\left[\beta_{\tau}\right]=\tau$ and that a smooth and regular parametrisation of $\beta_{\tau}$ is actually contained in the class $\mathcal{C}_{\tau}$. The formula for the volume in the statement of the lemma is a direct consequence of Hotelling's theorem, see Proposition 1. The four arcs on the great circle each of length $\pi / 2$ connecting neighbouring points in $\left\{P_{1}, P_{2}, P_{3}, P_{4}\right\}$ are the shortest possible connections on $\mathbb{S}^{2}$ so that $L \geqq \frac{\pi}{2}$; hence $\mathscr{L}\left(\beta_{\tau}\right)=4 L \geqq 2 \pi$ with equality if and only if $\beta_{\tau}$ is the great circle, that is, $\tau=\Theta_{1}=1$.

We conclude this section by analysing how length and tube volume of solutions depend on the given thickness.

8 The circular arcs indeed have common tangent lines at the concatenation points $P_{i}$, $i=1,2,3,4$, since the tangent vectors of the two arcs meeting at, say, endpoint $P_{1}$ are both contained in the tangent plane $T_{P_{1}} \mathbb{S}^{2}$ both enclosing the same angle with $E \cap T_{P_{1}} \mathbb{S}^{2}$, such that the reflection across the line $E \cap T_{P_{1}} \mathbb{S}^{2}$ produces the common tangent line through $P_{1}$ in $T_{P_{1}} \mathbb{S}^{2}$. 

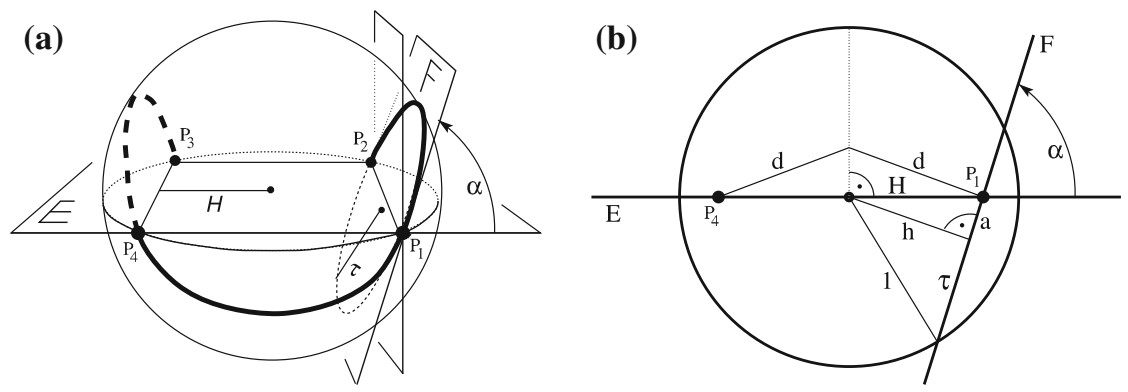

(c)
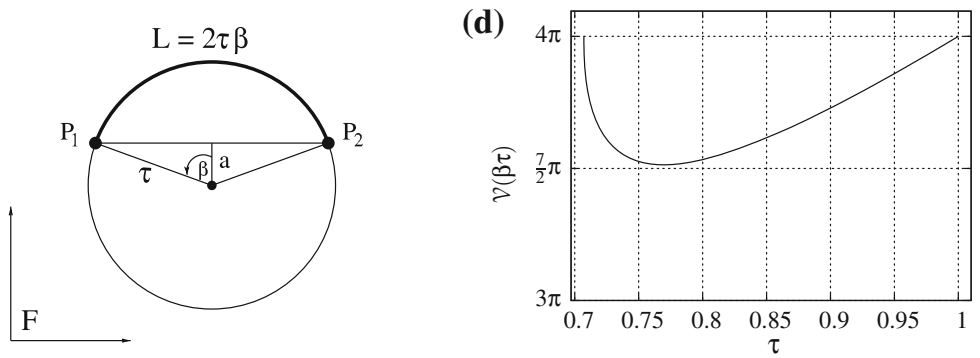

Fig. 8. a-c Determining the thickness of $\beta_{\tau}$ as described in Lemma 6. d The volume $\mathscr{V}$ of the tubular neighbourhood of $\beta_{\tau}$

Lemma 7. For given minimal thickness $\Theta \in(0,1]$, let $\gamma_{\Theta}$ be a solution of Problem $(\mathrm{P})$, and define the function $L:(0,1] \rightarrow[2 \pi, \infty)$ by $L(\Theta):=\mathscr{L}\left(\gamma_{\Theta}\right)$ and $V:(0,1] \rightarrow(0,4 \pi]$ by $V(\Theta):=\mathscr{V}\left(T_{\arcsin } \Theta\left(\gamma_{\Theta}\right)\right)$. Then

(i) L is a strictly decreasing function on $(0,1]$.

(ii) $L(1)=2 \pi$, and $L(\Theta) \rightarrow \infty$ as $\Theta \rightarrow+0$.

(iii) $\lim _{h \rightarrow+0} L(\Theta-h)=L(\Theta)$ for $\Theta \in(0,1]$.

(iv) $L(\Theta)=V(\Theta) /(2 \Theta)$ is differentiable at almost every $\Theta \in(0,1]$.

(v) $2 \pi \leqq L(\Theta) \leqq 2 \pi / \Theta$ and $4 \pi \Theta \leqq V(\Theta) \leqq 4 \pi$ for all $\Theta \in(0,1]$.

(vi) The functions $L$ and $V$ are upper semicontinuous on $(0,1]$.

Proof. (i) For $0<\Theta<\Theta^{\prime} \leqq 1$ and the corresponding solutions $\gamma_{\Theta} \in \mathcal{C}_{\Theta}$ and $\gamma_{\Theta^{\prime}} \in \mathcal{C}_{\Theta^{\prime}}$ for Problem (P) we have

$$
\triangle\left[\gamma_{\Theta^{\prime}}\right] \geqq \Theta^{\prime}>\Theta
$$

so that $\gamma_{\Theta^{\prime}} \in \mathcal{C}_{\Theta}$ as well. Hence $L(\Theta)=\mathscr{L}\left(\gamma_{\Theta}\right) \geqq \mathscr{L}\left(\gamma_{\Theta^{\prime}}\right)=L\left(\Theta^{\prime}\right)$, which proves that $L$ is a decreasing function. If $0<\Theta<\Theta^{\prime}<1$ we know that $\gamma_{\Theta^{\prime}}$ is not the great circle, and we may assume that $\gamma_{\Theta^{\prime}}$ has constant speed $v=\left|\gamma_{\Theta^{\prime}}\right|$ on $\mathbb{S}^{1}$. Recalling the arguments in the proof of Theorem 5 , there must be a function $\psi \in C_{0}^{\infty}\left(\mathbb{S}^{1}, \mathbb{R}^{3}\right)$ such that

$$
\delta \mathscr{L}\left(\gamma_{\Theta^{\prime}}, \psi\right):=\frac{d}{d \epsilon}\left[\mathscr{L}\left(\frac{\gamma_{\Theta^{\prime}}+\epsilon \psi}{\left|\gamma_{\Theta^{\prime}}+\epsilon \psi\right|}\right)\right]_{\epsilon=0} \neq 0,
$$


and since the first variation $\delta \mathscr{L}\left(\gamma_{\Theta^{\prime}}, \cdot\right)$ is a linear functional, we may assume that

$$
\delta \mathscr{L}\left(\gamma_{\Theta^{\prime}}, \psi\right)=1
$$

Notice that (3.23) and the claim established in the proof of Theorem 5 imply

$$
\Delta\left[\frac{\gamma_{\Theta^{\prime}}+\epsilon \psi}{\left|\gamma_{\Theta^{\prime}}+\epsilon \psi\right|}\right]>\Theta, \text { hence } \frac{\gamma_{\Theta^{\prime}}+\epsilon \psi}{\left|\gamma_{\Theta^{\prime}}+\epsilon \psi\right|} \in \mathcal{C}_{\Theta}
$$

for $\epsilon$ sufficiently small. One can check that there is an $\epsilon_{0}=\epsilon_{0}(\psi)>0$ such that $\frac{d^{2}}{d \epsilon^{2}}\left[\mathscr{L}\left(\frac{\gamma_{\Theta^{\prime}}+\epsilon \psi}{\left|\gamma_{\Theta^{\prime}}+\epsilon \psi\right|}\right)\right]$ is bounded uniformly in $\epsilon \in\left[-\epsilon_{0}, \epsilon_{0}\right]$, so that

$$
\mathscr{L}\left(\frac{\gamma_{\Theta^{\prime}}+\epsilon \psi}{\left|\gamma_{\Theta^{\prime}}+\epsilon \psi\right|}\right)=\mathscr{L}\left(\gamma_{\Theta^{\prime}}\right)+\epsilon \delta \mathscr{L}\left(\gamma_{\Theta^{\prime}}, \psi\right)+O\left(\epsilon^{2}\right) .
$$

Hence by virtue of (3.24) $\mathscr{L}\left(\frac{\gamma_{\Theta^{\prime}}+\epsilon \psi}{\left|\gamma_{\Theta^{\prime}}+\epsilon \psi\right|}\right)>\mathscr{L}\left(\gamma_{\Theta^{\prime}}\right)$ for $0<\epsilon \ll 1$, which implies by (3.25)

$$
L(\Theta)=\mathscr{L}\left(\gamma_{\Theta}\right) \underset{(3.25)}{\geqq} \mathscr{L}\left(\frac{\gamma_{\Theta^{\prime}}+\epsilon \psi}{\left|\gamma_{\Theta^{\prime}}+\epsilon \psi\right|}\right)>\mathscr{L}\left(\gamma_{\Theta^{\prime}}\right)=L\left(\Theta^{\prime}\right)
$$

for all $0<\Theta<\Theta^{\prime}<1$. If $\Theta<\Theta^{\prime}=1$ we find $\Theta^{\prime \prime} \in\left(\Theta, \Theta^{\prime}\right)$, so that according to what we have just proved and the monotonicity observed in the beginning

$$
L(\Theta)>L\left(\Theta^{\prime \prime}\right) \geqq L\left(\Theta^{\prime}\right),
$$

which finishes the proof of Part (i).

(ii) For the explicit solutions $\beta^{n, k} \in \mathcal{C}_{\Theta_{n}}$ constructed in Theorem 2 we have $\mathscr{V}\left(T_{\arcsin \Theta_{n}}\left(\beta^{n, k}\right)\right)=4 \pi$, and therefore by Proposition 1

$$
L\left(\Theta_{n}\right)=\mathscr{L}\left(\beta^{n, k}\right)=\frac{\mathscr{V}\left(T_{\arcsin \Theta_{n}}\left(\beta^{n, k}\right)\right)}{2 \Theta_{n}}=\frac{2 \pi}{\Theta_{n}}=\frac{2 \pi}{\sin \frac{\pi}{2 n}} \rightarrow \infty \text { as } n \rightarrow \infty .
$$

This together with the strict monotonicity shown in Part (i) establishes $L(\Theta) \rightarrow \infty$ as $\Theta \rightarrow+0$.

(iii) We consider the set of solutions $\gamma_{\Theta-h} \in \mathcal{C}_{\Theta-h}$ for $0<h<\Theta / 2$ and assume that all these curves have constant speed $v_{h}:=\left|\gamma_{\Theta-h}^{\prime}\right|>0$. As in (3.20) in the proof of Theorem 1 we use [13, Lemma 2] to obtain the uniform upper bound

$$
\left\|\Gamma_{h}\right\|_{C^{1,1}\left([0, L(\Theta-h)], \mathbb{R}^{3}\right)} \leqq 2+\frac{1}{\Theta-h}<2+\frac{2}{\Theta} \quad \text { for all } h \in(0, \Theta / 2)
$$

for the respective arc-length parametrisations $\Gamma_{h}:[0, L(\Theta-h)] \rightarrow \mathbb{R}^{3}$ of $\gamma_{\Theta-h}$. In addition, Lemma 5 applied to $\gamma:=\gamma_{\Theta-h} \in \mathcal{C}_{\Theta-h}$ implies

$$
\frac{\Theta}{2}<\Theta-h \leqq\left|\gamma_{\Theta-h}^{\prime}\right|=v_{h} \leqq \frac{1}{\Theta-h}<\frac{2}{\Theta} \quad \text { for all } h \in(0, \Theta / 2),
$$


so that one obtains, similarly as in (3.21),

$$
\begin{aligned}
\left\|\gamma_{\Theta-h}\right\|_{C^{1,1}\left(\mathbb{S}^{1}, \mathbb{R}^{3}\right)} & \leqq\left\|\gamma_{\Theta-h}\right\|_{C^{0}\left(\mathbb{S}^{1}, \mathbb{R}^{3}\right)}+v_{h}\left\|\Gamma_{h}^{\prime}\right\|_{C^{0}\left(\left[0, L(\Theta-h], \mathbb{R}^{3}\right)\right.}+\frac{v_{h}^{2}}{\Theta-h} \\
& \leqq 1+\frac{2}{\Theta}+\frac{8}{\Theta^{3}} \quad \text { for all } h \in(0, \Theta / 2) .
\end{aligned}
$$

The theorem of Arzelà-Ascoli yields a subsequence $h_{i} \rightarrow+0$ and a curve $\gamma \in$ $C^{1,1}\left(\mathbb{S}^{1}, \mathbb{R}^{3}\right)$ such that $\gamma_{\Theta-h_{i}} \rightarrow \gamma$ in $C^{1}\left(\mathbb{S}^{1}, \mathbb{R}^{3}\right)$ as $i \rightarrow \infty$, so that $\gamma\left(\mathbb{S}^{1}\right) \subset \mathbb{S}^{2}$ and $\Delta[\gamma] \geqq \Theta-\epsilon$ for each $\epsilon>0$ (and hence $\Delta[\gamma] \geqq \Theta$ ) by [20, Lemma 4] and [11, Lemma 5]. Moreover, by (3.26) $v:=\left|\gamma^{\prime}\right| \geqq \Theta / 2>0$ so that $\gamma \in \mathcal{C}_{\Theta}$, hence $\mathscr{L}(\gamma) \leqq \mathscr{L}\left(\gamma_{\Theta}\right)=L(\Theta)$. On the other hand, $\mathscr{L}$ is continuous in the $C^{1}$-topology, that is, from the monotonicity shown in Part (i) we infer

$$
L\left(\Theta-h_{l}\right)=\mathscr{L}\left(\gamma_{\Theta-h_{l}}\right) \stackrel{l \rightarrow \infty}{\longrightarrow} \mathscr{L}(\gamma) \leqq L(\Theta) \underset{(\mathrm{i})}{L} L\left(\Theta-h_{i}\right) \quad \text { for all } i \in \mathbb{N}
$$

which proves $\lim _{i \rightarrow \infty} L\left(\Theta-h_{i}\right)=\mathscr{L}(\gamma)=L(\Theta)$. In particular, $\gamma \in \mathcal{C}_{\Theta}$ is also a solution to Problem (P).

By the subsequence principle, we finally conclude $\lim _{h \rightarrow+0} L(\Theta-h)=L(\Theta)$. (Notice however, that a different subsequence $\tilde{h}_{i} \rightarrow+0$ could lead to a different solution $\tilde{\gamma} \neq \gamma$ in the $C^{1}$-limit $\gamma_{\Theta-\tilde{h}_{i}} \rightarrow \tilde{\gamma} \in \mathcal{C}_{\Theta}$ with $\mathscr{L}(\tilde{\gamma})=\mathscr{L}(\gamma)=L(\Theta)$.)

(iv) Proposition 1 applied to the solution $\gamma_{\Theta} \in \mathcal{C}_{\Theta}$ of Problem (P) gives

$$
L(\Theta)=\mathscr{L}\left(\gamma_{\Theta}\right) \underset{\text { Proposition 1 }}{=} \frac{\mathscr{V}\left(T_{\arcsin \Theta}\left(\gamma_{\Theta}\right)\right)}{2 \Theta}=\frac{V(\Theta)}{2 \Theta} .
$$

Since $L:(0,1] \rightarrow[2 \pi, \infty)$ is (strictly) monotone, it is differentiable almost everywhere on $(0,1]$.

(v) By Proposition 1 we have for all $\Theta \in(0,1]$

$$
L(\Theta)=\mathscr{L}\left(\gamma_{\Theta}\right) \underset{\text { Proposition } 1}{=} \frac{\mathscr{V}\left(T_{\arcsin \Theta}\left(\gamma_{\Theta}\right)\right)}{2 \Theta} \leqq \frac{4 \pi}{2 \Theta}=\frac{2 \pi}{\Theta} .
$$

On the other hand, the great circle $c_{g} \in \mathcal{C}_{1} \subset \mathcal{C}_{\Theta}$ has length $2 \pi=\mathscr{L}\left(c_{g}\right) \leqq$ $\mathscr{L}\left(\gamma_{\Theta}\right)=L(\Theta)$ for all $\Theta \in(0,1]$. The corresponding inequality for the volume $V(\Theta)$ follows now from Part (iv).

(vi) For $\Theta_{i} \rightarrow \Theta \in(0,1]$ as $i \rightarrow \infty$ consider a subsequence $\left\{\Theta_{j}\right\} \subset\left\{\Theta_{i}\right\}$ such that $L\left(\Theta_{j}\right) \rightarrow \lim \sup _{i \rightarrow \infty} L\left(\Theta_{i}\right)$ as $j \rightarrow \infty$. If there are infinitely many $j$ such that $\Theta_{j} \leqq \Theta$, then we obtain from Part (iii) $\lim _{j} \sup _{i \rightarrow \infty} L\left(\Theta_{i}\right)=$ $\lim _{j \rightarrow \infty} L\left(\Theta_{j}\right)=L(\Theta)$. On the other hand, for all $j$ with $\Theta_{j}>\Theta$ we have, by Part (i), $L(\Theta)>L\left(\Theta_{j}\right)$ such that $L(\Theta) \geqq \lim _{j \rightarrow \infty} L\left(\Theta_{j}\right)=\lim _{\sup _{i \rightarrow \infty}} L\left(\Theta_{i}\right)$. Part (iv) implies

$$
\limsup _{i \rightarrow \infty} V\left(\Theta_{i}\right) \underset{\text { (iv) }}{=} \limsup _{i \rightarrow \infty} 2 \Theta_{i} L\left(\Theta_{i}\right) \leqq 2 \Theta L(\Theta) \underset{\text { (iv) }}{=} V(\Theta) .
$$

The lower bound for the volume $V(\Theta)=\mathscr{V}\left(T_{\arcsin \Theta}\left(\gamma_{\Theta}\right)\right)$ depicted in Fig. 4 improves the lower estimate in Lemma 7 (v) considerably, and is established in 
Lemma 8. (Lower volume bound for solutions) The function $V:(0,1] \rightarrow(0,4 \pi]$ is differentiable almost everywhere on $(0,1]$ and satisfies the estimate

$$
\frac{4 \pi}{\Theta_{n}} \Theta \leqq V(\Theta) \leqq 4 \pi \quad \text { for all } \Theta \leqq \Theta_{n}=\sin \frac{\pi}{2 n}, n \in \mathbb{N}
$$

In particular, $4 \pi \geqq V(\Theta) \geqq 4 \pi \Theta / \Theta_{n}$ for $\Theta \in\left(\Theta_{n+1}, \Theta_{n}\right], n \in \mathbb{N}$.

Notice that we can use Proposition 1 twice to interpret the lower bound for $V(\Theta)$ as the volume of the tube $T_{\arcsin \Theta}\left(\beta^{n, k}\right)$. In fact, for $\vartheta:=\arcsin \Theta$ one has

$$
\mathscr{V}\left(T_{\vartheta}\left(\beta^{n, k}\right)\right)_{\text {Proposition 1 }}^{=} 2 \Theta \mathscr{L}\left(\beta^{n, k}\right) \underset{\text { Proposition 1 }}{=} 2 \Theta \frac{\mathscr{V}\left(T_{\vartheta_{n}}\left(\beta^{n, k}\right)\right)}{2 \Theta_{n}}=\frac{4 \pi \Theta}{\Theta_{n}} .
$$

Proof. Combining Parts (i) and (iv) of Lemma 7 we obtain that $V$ is differentiable almost everywhere and satisfies

$$
\begin{aligned}
0 \geqq & L^{\prime}(\Theta)=\left(\frac{V(\Theta)}{2 \Theta}\right)^{\prime}=-\frac{1}{2 \Theta^{2}} V(\Theta) \\
& +\frac{1}{2 \Theta} V^{\prime}(\Theta) \quad \text { for almost everywhere } \Theta \in(0,1],
\end{aligned}
$$

that is,

$$
\frac{1}{2 \Theta} V^{\prime}(\Theta) \leqq \frac{1}{2 \Theta^{2}} V(\Theta) \quad \text { for almost everywhere } \Theta \in(0,1]
$$

Since $V(\Theta) \geqq 4 \pi \Theta>0$ for all $\Theta \in(0,1]$ by Lemma 7 (v), we conclude

$$
(\log V(\Theta))^{\prime}=\frac{V^{\prime}(\Theta)}{V(\Theta)} \leqq \frac{1}{\Theta} \quad \text { for almost everywhere } \Theta \in(0,1] .
$$

Integrating this inequality on $\left[\Theta, \Theta_{n}\right]$ for $\Theta \in\left(0, \Theta_{n}\right), n \in \mathbb{N}$, we obtain with $V\left(\Theta_{n}\right)=4 \pi$

$\log 4 \pi-\log V(\Theta)=\log V\left(\Theta_{n}\right)-\log V(\Theta) \leqq \int_{\Theta}^{\Theta_{n}} \frac{1}{\Theta} \mathrm{d} \Theta=\log \Theta_{n}-\log \Theta$,

hence $\frac{4 \pi}{\Theta_{n}} \Theta \leqq V(\Theta)$.

Corollary 2. $V(\Theta) \rightarrow 4 \pi$ as $\Theta \rightarrow+0$.

Proof. For $\Theta \in\left(\Theta_{n+1}, \Theta_{n}\right]$ we have

$$
V(\Theta) \geqq \frac{4 \pi \Theta}{\Theta_{n}}>\frac{4 \pi \Theta_{n+1}}{\Theta_{n}}=4 \pi \frac{\sin \frac{\pi}{2 n+2}}{\sin \frac{\pi}{2 n}} \stackrel{n \rightarrow \infty}{\longrightarrow} 4 \pi .
$$

This asymptotic behaviour of the volume confirms our intuition that it is easier to cover the sphere with thin ropes than with thick ones-there is simply more freedom with long and thin ropes to "fill" the gaps on the surface of the unit sphere. 


\section{Uniqueness}

In the following we are going to work with geodesic balls $\mathscr{B}_{\vartheta}(\xi):=\left\{\eta \in \mathbb{S}^{2}\right.$ : $\left.\operatorname{dist}_{\mathbb{S}^{2}}(\eta, \xi)<\vartheta\right\}$. on the unit sphere. Furthermore, we will use the notation

$$
\begin{aligned}
\overline{\mathscr{B}_{\vartheta}(\xi)} & :=\left\{\eta \in \mathbb{S}^{2}: \operatorname{dist}_{\mathbb{S}^{2}}(\eta, \xi) \leqq \vartheta\right\}, \\
\partial \mathscr{B}_{\vartheta}(\xi) & :=\left\{\eta \in \mathbb{S}^{2}: \operatorname{dist}_{\mathbb{S}^{2}}(\eta, \xi)=\vartheta\right\} .
\end{aligned}
$$

Proposition 2. Let $\gamma: \mathbb{S}^{1} \rightarrow \mathbb{S}^{2}$ be a closed, rectifiable and continuous curve with positive thickness $\Theta:=\triangle[\gamma]>0, \vartheta:=\arcsin \Theta \leqq \pi / 2$, and $\xi \in \mathbb{S}^{2}$ such that

$$
\gamma\left(\mathbb{S}^{1}\right) \cap \mathscr{B}_{\vartheta}(\xi)=\varnothing .
$$

If there are two points $P, Q \in \partial \mathscr{B}_{\vartheta}(\xi) \cap \gamma\left(\mathbb{S}^{1}\right)$ with $0<\operatorname{dist}_{\mathbb{S}^{2}}(P, Q)<2 \vartheta$, then the shorter circular sub-arc of the geodesic circle $\partial \mathscr{B}_{\vartheta}(\xi)$ connecting $P$ and $Q$ is contained in $\gamma\left(\mathbb{S}^{1}\right)$.

Proof. Recall from [13, Lemma 2] that the arc-length parametrisation $\Gamma: S_{L} \rightarrow$ $\mathbb{S}^{2}$ of $\gamma$ (with length $L:=\mathscr{L}(\gamma)>0$ ) is of class $C^{1,1}\left(S_{L}, \mathbb{R}^{3}\right)$, which allows us in the following to speak of tangential properties of $\gamma$.

We set

$$
\begin{aligned}
& \vartheta_{0}:=\vartheta, \vartheta_{1}:=\frac{1}{2} \operatorname{dist}_{\mathbb{S}^{2}}(P, Q)<\vartheta_{0}, \\
& \xi_{0}:=\xi, \quad \xi_{1}:=c_{g}\left(\vartheta_{1}\right),
\end{aligned}
$$

where $c_{g}:[0,2 \pi] \rightarrow \mathbb{S}^{2}$ denotes the unit speed parametrisation of the great circle through $P$ and $Q$ with $c_{g}(0)=P$ and $c_{g}\left(2 \vartheta_{1}\right)=Q$. Thus $P$ and $Q$ are antipodal points on the geodesic circle $\partial \mathscr{B}_{\vartheta_{1}}\left(\xi_{1}\right)$. Since by (4.1) $\gamma$ is tangent to the geodesic circle $\partial \mathscr{B}_{\vartheta_{0}}\left(\xi_{0}\right)$ at $P$ and $Q$, it cannot be tangent to $\partial \mathscr{B}_{\vartheta_{1}}\left(\xi_{1}\right)$ at $P$ or $Q$. Hence $\mathscr{B}_{\vartheta_{1}}\left(\xi_{1}\right) \cap \gamma\left(\mathbb{S}^{1}\right) \neq \emptyset$, and we consider the family of geodesic balls $\mathscr{B}_{\vartheta_{s}}\left(\xi_{s}\right)$ defined by

$$
\xi_{s}:=\tilde{c}_{g}(s) \quad \text { and } \quad \vartheta_{s}:=\left|P-\xi_{s}\right| \text { for } s \in[0,1],
$$

where $\tilde{c}_{g}:\left[0,2 \pi / \operatorname{dist}_{\mathbb{S}^{2}}\left(\xi_{0}, \xi_{1}\right)\right] \rightarrow \mathbb{S}^{2}$ is the constant speed parametrisation of the great circle through $\xi_{0}$ and $\xi_{1}$ with $\tilde{c}_{g}(0)=\xi_{0}, \tilde{c}_{g}(1)=\xi_{1}$, and $\left|\tilde{c}_{g}{ }^{\prime}\right| \equiv$ $\operatorname{dist}_{\mathbb{S}^{2}}\left(\xi_{0}, \xi_{1}\right)$. We notice that $\vartheta_{s}<\vartheta_{0}$ for all $s \in(0,1]$, and claim that

$$
\partial \mathscr{B}_{\vartheta_{s}}\left(\xi_{s}\right) \cap \gamma\left(\mathbb{S}^{1}\right)=\{P, Q\} \quad \text { for all } s \in(0,1] .
$$

Indeed, otherwise we would have (at least) three points of the curve on a geodesic circle $\partial \mathscr{B}_{\vartheta^{*}}\left(\xi_{s^{*}}\right)$ for some $s^{*} \in(0,1]$, which is a Euclidean circle of (Euclidean) radius $\sin \vartheta_{s^{*}}<\sin \vartheta_{0}=\Theta=\Delta[\gamma]$. This, however, contradicts the definition of $\triangle[\gamma]$ in (1.1).

By (4.1) $\gamma$ is tangent to $\partial \mathscr{B}_{\vartheta_{0}}\left(\xi_{0}\right)$ at $P$ and $Q$, and since the circular arcs $\partial \mathscr{B}_{\vartheta_{s}}\left(\xi_{s}\right)$ sweep out the open region $\mathscr{B}_{\vartheta_{1}}\left(\xi_{1}\right) \backslash \overline{\mathscr{B}_{\vartheta_{0}}\left(\xi_{0}\right)}$, that is,

$$
\mathscr{B}_{\vartheta_{1}}\left(\xi_{1}\right) \backslash \overline{\mathscr{B}_{\vartheta_{0}}\left(\xi_{0}\right)} \subset \bigcup_{s \in(0,1]} \partial \mathscr{B}_{\vartheta_{s}}\left(\xi_{s}\right) \backslash \overline{\mathscr{B}_{\vartheta_{0}}\left(\xi_{0}\right)},
$$


we conclude from (4.2) that the shorter sub-arc $\gamma_{1} \subset \gamma$ connecting $P$ and $Q$ must be equal to the shorter circular arc on $\partial \mathscr{B}_{\vartheta_{0}}\left(\xi_{0}\right)=\partial \mathscr{B}_{\vartheta}(\xi)$ with endpoints $P$ and $Q$.

Remark 2. An analogous statement also holds for closed space curves $\gamma: \mathbb{S}^{1} \rightarrow$ $\mathbb{R}^{3}$ with $\Theta:=\triangle[\gamma]>0[8$, Satz 3.27]: If $\gamma$ has empty intersection with a Euclidean ball $B_{\Theta}(x)$, such that the boundary $\partial B_{\Theta}(x)$ contains two non-antipodal curve points $P$ and $Q$, then the shorter sub-arc of the great circle connecting $P$ and $Q$ on $\partial B_{\Theta}(x)$ is contained in $\gamma\left(\mathbb{S}^{1}\right)$. The fact that $\gamma$ connects $P$ and $Q$ within the sphere $\partial B_{\Theta}(x)$ can be proven with an argument similar to that in the proof of Proposition 2. This connecting arc $\gamma_{P Q}$ lies, indeed, on a great circle, because any triple of distinct curve points $x, y$, and $z$ on $\gamma_{P Q}$ span a plane whose intersection with $B_{\Theta}(x)$ is a circle of radius, at most, $\Theta$. This implies, by Definition (1.1) of the thickness $\Delta[\gamma]=\Theta$, that this intersection circle must have radius $\Theta$, that is, is a great circle, which is uniquely determined by $x$ and $y$ alone, so that any other point $\tilde{z}$ distinct from $x, y$, and $z$ must lie on the same great circle.

Lemma 9. (Characterisation of sphere filling curves) For a closed rectifiable continuous curve $\gamma: \mathbb{S}^{1} \rightarrow \mathbb{S}^{2}$ with positive thickness $\Theta:=\Delta[\gamma]>0, \vartheta:=$ $\arcsin \Theta \leqq \pi / 2$, the following two statements are equivalent:

(i) $\mathscr{V}\left(T_{\vartheta}(\gamma)\right)=4 \pi$;

(ii) For any $\xi \in \mathbb{S}^{2}$ such that $\mathscr{B}_{\vartheta}(\xi) \cap \gamma\left(\mathbb{S}^{1}\right)=\emptyset$ one of the following is true:

(a) $\partial \mathscr{B}_{\vartheta}(\xi) \cap \gamma\left(\mathbb{S}^{1}\right)=\{P, Q\}$ with $\operatorname{dist}_{\mathbb{S}^{2}}(P, Q)=2 \vartheta$ (antipodal points);

(b) $\partial \mathscr{B}_{\vartheta}(\xi) \cap \gamma\left(\mathbb{S}^{1}\right)=\{$ semicircle of spherical radius $\vartheta\}$;

(c) $\partial \mathscr{B}_{\vartheta}(\xi) \cap \gamma\left(\mathbb{S}^{1}\right)=\partial \mathscr{B}_{\vartheta}(\xi)$.

Proof. (i) $\Rightarrow$ (ii). Let $\xi \in \mathbb{S}^{2}$ be a point such that the open geodesic ball $\mathscr{B}_{\vartheta}(\xi)$ has empty intersection with the curve $\gamma\left(\mathbb{S}^{1}\right)$. We claim that

$$
\mathscr{S}:=\partial \mathscr{B}_{\vartheta}(\xi) \cap \gamma\left(\mathbb{S}^{1}\right) \neq \emptyset .
$$

Indeed, otherwise we could infer $\delta:=\operatorname{dist}_{\mathbb{S}^{2}}\left(\xi, \gamma\left(\mathbb{S}^{1}\right)\right)-\vartheta>0$, so that $T_{\vartheta}(\gamma) \cap$ $\mathscr{B}_{\delta}(\xi)=\emptyset$, which implies $\mathscr{V}\left(T_{\vartheta}(\gamma)\right) \leqq \mathscr{V}\left(\mathbb{S}^{2} \backslash \mathscr{B}_{\delta}(\xi)\right)<4 \pi$, contradicting Assumption (i).

If the closed set $\mathscr{S}$ is contained in an open semicircle on $\partial \mathscr{B}_{\vartheta}(\xi)$, then we find two points $\eta, \zeta \in \mathscr{S}$ such that ${ }^{9}$

$$
0 \leqq \operatorname{dist}_{\mathbb{S}^{2}}(\eta, \zeta)=\max _{\mathscr{S} \times \mathscr{S}} \operatorname{dist}_{\mathbb{S}^{2}}(\cdot, \cdot)<2 \vartheta
$$

Applying Proposition 2 we infer that the whole shorter sub-arc of $\partial \mathscr{B} \vartheta(\xi)$ connecting $\eta$ and $\zeta$ is contained in $\mathscr{S}$, and is (by (4.3)) consequently equal to $\mathscr{S}$. On this circular arc we find a point $q \in \mathscr{S}$ with $\operatorname{dist}_{\mathbb{S}^{2}}(q, \eta)=\operatorname{dist}_{\mathbb{S}^{2}}(q, \zeta)$, which can be joined with the centre $\xi$ by the unique unit speed geodesic given by the great circle $c_{g}:[0,2 \pi] \rightarrow \mathbb{S}^{2}$ with $c_{g}(0)=q, c_{g}^{\prime}(0) \perp \mathscr{S}$, and $c_{g}(\vartheta)=\xi$.

${ }^{9}$ We also allow the coincidence $\eta=\zeta$ (in which case $\mathscr{S}=\{\eta\}$ ) at this stage. 
We claim that there is a small number $\epsilon>0$, such that

$$
\delta:=\operatorname{dist}_{\mathbb{S}^{2}}\left(c_{g}(\vartheta+\epsilon), \gamma\left(\mathbb{S}^{1}\right)\right)-\vartheta>0,
$$

which would imply that $T_{\vartheta}(\gamma) \cap \mathscr{B}_{\delta}\left(c_{g}(\vartheta+\epsilon)\right)=\emptyset$, hence $\mathscr{V}\left(T_{\vartheta}(\gamma)\right) \leqq$ $\mathscr{V}\left(\mathbb{S}^{2} \backslash \mathscr{B}_{\delta}\left(c_{g}(\vartheta+\epsilon)\right)\right)<4 \pi$ contradicting Assumption (i) and therefore ruling out the situation that $\mathscr{S}$ is contained in any open semicircle.

To prove the claim, we suppose to the contrary that there is a sequence of curve points $p_{n} \in \gamma\left(\mathbb{S}^{1}\right)$ such that

$$
\operatorname{dist}_{\mathbb{S}^{2}}\left(c_{g}(\vartheta+1 / n), \gamma\left(\mathbb{S}^{1}\right)\right)=\operatorname{dist}_{\mathbb{S}^{2}}\left(c_{g}(\vartheta+1 / n), p_{n}\right) \leqq \vartheta \quad \text { for all } n \in \mathbb{N} \text {. }
$$

Since $\gamma\left(\mathbb{S}^{1}\right)$ is compact we may assume that $p_{n} \rightarrow p \in \gamma\left(\mathbb{S}^{1}\right)$ as $n \rightarrow \infty$. From $\mathscr{B}_{\vartheta}(\xi) \cap \gamma\left(\mathbb{S}^{1}\right)=\emptyset$ we infer from (4.4) as $n \rightarrow \infty$

$$
\begin{aligned}
\vartheta & \leqq \operatorname{dist}_{\mathbb{S}^{2}}(\xi, p)=\operatorname{dist}_{\mathbb{S}^{2}}\left(c_{g}(\vartheta), p\right)=\lim _{n \rightarrow \infty} \operatorname{dist}_{\mathbb{S}^{2}}\left(c_{g}(\vartheta+1 / n), p_{n}\right) \leqq \vartheta, \text { that is } \\
p & \in \partial \mathscr{B}_{\vartheta}(\xi) \cap \gamma\left(\mathbb{S}^{1}\right)=\mathscr{S} .
\end{aligned}
$$

On the other hand, one has

$$
\operatorname{dist}_{\mathbb{S}^{2}}(x, \mathscr{S}) \geqq\left|\frac{\eta+\zeta}{2}-\xi\right|=: c(\eta, \zeta)>0
$$

for all $x \in \overline{\mathscr{B}_{\vartheta}\left(c_{g}(\vartheta+1 / n)\right)} \backslash \mathscr{B}_{\vartheta}(\xi)$, since the circular arc $\mathscr{S}$ (with endpoints $\eta$ and $\zeta)$ is strictly shorter than $\pi \vartheta$. Consequently, $\operatorname{dist}_{\mathbb{S}^{2}}\left(p_{n}, \mathscr{S}\right) \geqq c(\eta, \zeta)>0$ for all $n \in \mathbb{N}$, and hence $\operatorname{dist}_{\mathbb{S}^{2}}(p, \mathscr{S}) \geqq c(\eta, \zeta)>0$ contradicting (4.5), which proves the claim.

Since we know now that $\mathscr{S}$ is not contained in any open semicircle on $\partial \mathscr{B}_{\vartheta}(\xi)$, we know that $\sharp \mathscr{S} \geqq 3$ unless $\mathscr{S}$ consists precisely of two antipodal points, which is case (a).

If $\mathscr{S}$ is contained in a closed semicircle, it must contain the endpoints $p_{1}$, $p_{2}$ of that semicircle (otherwise it would be contained in a different open semicircle, which was excluded above). Since $\sharp \mathscr{S} \geqq 3$ we find (at least) one point $q \in \mathscr{S} \backslash\left\{p_{1}, p_{2}\right\}$ so that $\operatorname{dist}_{\mathbb{S}^{2}}\left(p_{1}, q\right)<2 \vartheta$ and $\operatorname{dist}_{\mathbb{S}^{2}}\left(q, p_{2}\right)<2 \vartheta$. Consequently, we can apply Proposition 2 to find that $\mathscr{S}$ equals the closed semicircle with endpoints $p_{1}$ and $p_{2}$, which is case (b).

Finally, we have to deal with the situation that $\mathscr{S}$ is not contained in any closed semicircle. Consider $q \in \mathscr{S}$ and its antipodal point $q^{\prime} \in \partial \mathscr{B}_{\vartheta}(\xi)$.

If $q^{\prime} \in \mathscr{S}$, then we find on each of the two open semicircles $C_{1}, C_{2} \subset \partial \mathscr{B}_{\vartheta}(\xi)$ bounded by $q$ and $q^{\prime}$ (at least) one point of $\mathscr{S}$, say $p_{1} \in \mathscr{S} \cap C_{1}$ and $p_{2} \in \mathscr{S} \cap C_{2}$. Otherwise $\mathscr{S}$ would be contained in one of the two closed semicircles $C_{i} \cup\left\{q, q^{\prime}\right\}$, $i=1,2$.

Since $p_{1}$ and $p_{2}$ are not antipodal to $q$ or $q^{\prime}$, we can apply Proposition 2 to connect $q$ and $q^{\prime}$ with $p_{1}$ and $p_{2}$ by circular arcs contained in $\mathscr{S}$, which proves $\mathscr{S}=\partial \mathscr{B}_{\vartheta}(\xi)$, that is, we are in situation (c).

If, on the other hand, $q^{\prime} \notin \mathscr{S}$ we have $\operatorname{dist}_{\mathbb{S}^{2}}\left(q^{\prime}, \mathscr{S}\right)>0$, and we can take the largest open circular arc $C$ on $\partial \mathscr{B}_{\vartheta}(\xi) \backslash \mathscr{S}$ containing $q^{\prime}$. By definition, this arc has 
endpoints $p_{1}, p_{2} \in \mathscr{S}$, and $C$ is strictly shorter than a semicircle, since otherwise $\mathscr{S}$ would be contained in a closed semicircle. Thus we can apply Proposition 2 to $p_{1}$ and $p_{2}$, to $p_{1}$ and $q$, and to $p_{2}$ and $q$, respectively, to find that the circular arcs on $\partial \mathscr{B}_{\vartheta}(\xi)$ connecting $p_{1}$ and $p_{2}, p_{1}$ and $q$, and $p_{2}$ and $q$, are contained in $\mathscr{S}$, which proves $\mathscr{S}=\partial \mathscr{B}_{\vartheta}(\xi)$ also in this case. So we are in situation (c) again.

(ii) $\Rightarrow$ (i). For any $\xi \in \mathbb{S}^{2}$ with $\operatorname{dist}_{\mathbb{S}^{2}}\left(\xi, \gamma\left(\mathbb{S}^{1}\right)\right) \geqq \vartheta$ and with (a), or (b), or (c) we infer the existence of a point $p_{\xi} \in \gamma\left(\mathbb{S}^{1}\right)$ with $\operatorname{dist}_{\mathbb{S}^{2}}\left(\xi, p_{\xi}\right)=\vartheta$. Hence $\mathbb{S}^{2} \subset \overline{T_{\vartheta}(\gamma)} \subset \mathbb{S}^{2}$, which implies $4 \pi=\mathscr{V}\left(\overline{T_{\vartheta}(\gamma)}\right)=\mathscr{V}\left(T_{\vartheta}(\gamma)\right)$.

Lemma 10. (Characteristic patterns of sphere filling curves I) Let $\gamma: \mathbb{S}^{1} \rightarrow \mathbb{S}^{2}$ be a closed rectifiable continuous curve with positive thickness $\Theta:=\Delta[\gamma]>0$, $\vartheta:=\arcsin \Theta \leqq \pi / 2$ such that $\mathscr{V}\left(T_{\vartheta}(\gamma)\right)=4 \pi$. Suppose that there is a plane $E \subset \mathbb{R}^{3}$ containing $0 \in \mathbb{R}^{3}$ such that $k:=\sharp\left(E \cap \gamma\left(\mathbb{S}^{1}\right)\right)<\infty$. Moreover, assume that the intersection $\left\{p_{1}, \ldots, p_{k}\right\}:=E \cap \gamma\left(\mathbb{S}^{1}\right)$ satisfies

(i) $\operatorname{dist}_{\mathbb{S}^{2}}\left(p_{l}, p_{l+1}\right)=2 \vartheta$ for all $l=1, \ldots, k$, where we set $p_{k+1}:=p_{1}$.

(ii) $\gamma$ intersects $E$ orthogonally at each point $p_{l}, l=1, \ldots, k$.

Then $k=2 n$ for some $n \in \mathbb{N}$, and $\gamma\left(\mathbb{S}^{1}\right)$ contains a semicircle of (Euclidean) radius $\Theta$ in each of the two half-spaces bounded by $E$.

Proof. The positive thickness $\Theta$ guarantees that $\gamma$ is simple, and since $\gamma$ is also closed, we find that $k$ is even and write $k=: 2 n$ for some $n \in \mathbb{N}$.

$E$ cuts the sphere $\mathbb{S}^{2}$ into two hemispheres $\mathbb{S}^{w}$ and $\mathbb{S}^{e}$ (both taken to be relatively closed in $\mathbb{S}^{2}$ ). It suffices to give the argument for $\mathbb{S}^{w}$.

Every intersection point $p_{l} \in E \cap \gamma\left(\mathbb{S}^{1}\right)$ is connected by the curve $\gamma$ within $\mathbb{S}^{w}$ to some other intersection point $p_{m} \in E \cap \gamma\left(\mathbb{S}^{1}\right), l \neq m$. Since $\mathbb{S}^{w}$ is homeomorphic to a flat disk and $\gamma$ is simple, we find two distinct points $p_{i}, p_{j} \in E \cap \gamma\left(\mathbb{S}^{1}\right)$ with $\operatorname{dist}_{\mathbb{S}^{2}}\left(p_{i}, p_{j}\right)=2 \vartheta$ such that the closed sub-arc $\tilde{\gamma} \subset \mathbb{S}^{w} \cap \gamma$ connecting $p_{i}$ and $p_{j}$ within $\mathbb{S}^{w}$ satisfies $\tilde{\gamma} \cap E=\left\{p_{i}, p_{j}\right\}$.

We consider the geodesic ball $\mathscr{B}_{\vartheta}(\xi)$ that contains $p_{i}$ and $p_{j}$ as antipodal points in its boundary $\partial \mathscr{B}_{\vartheta}(\xi)$. Since $\gamma$ intersects $E$ orthogonally in $p_{i}$ and $p_{j}$ it is tangent to $\partial \mathscr{B}_{\vartheta}(\xi)$ in $p_{i}$ and $p_{j}$. The spherical torus property (ST) (see Remark 1) implies that

$$
\gamma\left(\mathbb{S}^{1}\right) \cap \mathscr{B}_{\vartheta}(\xi)=\emptyset .
$$

If $\tilde{\gamma} \cap \partial \mathscr{B}_{\vartheta}(\xi) \backslash\left\{p_{i}, p_{j}\right\} \neq \emptyset$ then there is (at least) one point $q \in \tilde{\gamma} \cap \partial \mathscr{B}_{\vartheta}(\xi)$ with $0<\operatorname{dist}_{\mathbb{S}^{2}}\left(q, p_{i}\right)<2 \vartheta$ and $0<\operatorname{dist}_{\mathbb{S}^{2}}\left(q, p_{j}\right)<2 \vartheta$, so that we can apply Proposition 2 to $p_{i}$ and $q$, and to $p_{j}$ and $q$, to find that $\gamma\left(\mathbb{S}^{1}\right)$ contains the closed semicircle $\partial \mathscr{B}_{\vartheta}(\xi) \cap \mathbb{S}^{w}$, which has Euclidean radius $\Theta=\sin \vartheta$, and we are done.

If $\tilde{\gamma} \cap \partial \mathscr{B}_{\vartheta}(\xi)=\left\{p_{i}, p_{j}\right\}$ we argue as follows. For an arbitrary $q \in \tilde{\gamma}$ we consider the unit speed geodesic $\eta_{q}:[0,2 \pi] \rightarrow \mathbb{S}^{2}$ starting in $q$ perpendicularly to $\tilde{\gamma}$, that is, with $\eta_{q}(0)=q, \eta_{q}^{\prime}(0) \perp \gamma$ at $q$, and $\left|\eta_{q}^{\prime}\right| \equiv 1$ on $[0,2 \pi]$, so that for $0<\epsilon \ll 1$, the point $\eta_{q}(\epsilon)$ is contained in the open spherical region $\mathscr{R} \subset \mathbb{S}^{2}$ bounded by the curve $\tilde{\gamma} \cup\left(\partial \mathscr{B}_{\vartheta}(\xi) \cap \mathbb{S}^{e}\right)$. Notice, first, that $\eta_{p_{i}}(2 \vartheta)=p_{j}$, $\eta_{p_{j}}(2 \vartheta)=p_{i}$, and also that $\mathscr{R} \cap \gamma\left(\mathbb{S}^{1}\right)=\emptyset$ by our choice of the points $p_{i}, p_{j}$, and by (4.6). 
We infer from the spherical torus property (ST), as before, that for $q \in \tilde{\gamma}$ we have

$$
\gamma\left(\mathbb{S}^{1}\right) \cap \mathscr{B}_{\vartheta}\left(\eta_{q}(\vartheta)\right)=\emptyset
$$

Relation (4.7) readily implies

$$
\mathscr{B} \vartheta\left(\eta_{q}(\vartheta)\right) \subset \mathscr{R} \quad \text { for all } q \in \tilde{\gamma} .
$$

Since $\mathscr{V}\left(T_{\vartheta}(\gamma)\right)=4 \pi$, we obtain by Lemma 9 either

$$
\partial \mathscr{B}_{\vartheta}\left(\eta_{q}(\vartheta)\right) \cap \gamma\left(\mathbb{S}^{1}\right)=\left\{q, \eta_{q}(2 \vartheta)\right\} \quad \text { (antipodal points), }
$$

a case which will be excluded later, or (in case (b) or (c) of Lemma 9) $\gamma\left(\mathbb{S}^{1}\right)$ contains a semicircle $S_{q}:=\partial \mathscr{B}_{\vartheta}\left(\eta_{q}(\vartheta)\right) \cap \gamma\left(\mathbb{S}^{1}\right)$ (containing $q$ ) of spherical radius $\vartheta$ and therefore of Euclidean radius $\Theta=\sin \vartheta$.

In that case, we have

$$
S_{q} \cap \mathbb{S}^{w} \subset \tilde{\gamma}
$$

because $\tilde{\gamma}$ is the connected sub-arc of $\gamma \cap \mathbb{S}^{w}$ containing the point $q$.

We claim that

$$
S_{q} \cap\left(\mathbb{S}^{e} \backslash E\right)=\emptyset,
$$

which means $S_{q} \subset \mathbb{S}^{w}$, which is the conclusion of the proof.

To show (4.11) we assume that there is some point $\tilde{q} \in S_{q} \cap\left(\mathbb{S}^{e} \backslash E\right)$, which implies that $p_{i} \in S_{q}$ or $p_{j} \in S_{q}$ by (4.10) and by connectivity of $S_{q}$ and $\tilde{\gamma}$ whose endpoints are $p_{i}$ and $p_{j}$. Relation (4.6) implies that $S_{q}$ is tangent to $\partial \mathscr{B}_{\vartheta}(\xi)$ at $p_{i}$ or $p_{j}$. For $S_{q}$ is a semicircle of spherical radius $\vartheta$ we have either $S_{q} \subset \partial \mathscr{B}_{\vartheta}(\xi)$ contradicting the fact that $q \notin \partial \mathscr{B}_{\vartheta}(\xi)$, or

$$
\partial \mathscr{B}_{\vartheta}(\xi) \cap \partial \mathscr{B}_{\vartheta}\left(\eta_{q}(\vartheta)\right)=\left\{p_{i}\right\} \quad \text { or }=\left\{p_{j}\right\} .
$$

In that case, we conclude with (4.7) that $\eta_{q}(\vartheta) \notin \mathscr{R}$ contradicting (4.8), which proves (4.11).

Finally, we need to exclude option (4.9) to finish the whole proof. Since positive thickness $\Theta=\Delta[\gamma]$ implies that the arc-length parametrisation of $\gamma$ is of class $C^{1,1}$, in particular that the tangent vector is continuous, we infer that the antipodal mapping $f: \tilde{\gamma} \longrightarrow \mathbb{S}^{2}, q \mapsto f(q):=\eta_{q}(2 \vartheta)$ is continuous. Moreover, $f(q) \in \tilde{\gamma}$ for all $q \in \tilde{\gamma}$, which can be seen as follows. According to (4.9) we have $f(q) \in \gamma\left(\mathbb{S}^{1}\right)$ which yields by (4.6) the relation $f(q) \notin \mathscr{B} \vartheta(\xi)$. This, together with (4.8), implies that $f(q) \in\left[\partial \mathscr{B}_{\vartheta}(\xi) \cap \mathbb{S}^{e}\right] \cup\left[\mathbb{S}^{w} \cap \overline{\mathscr{R}}\right]$, where $\overline{\mathscr{R}}$ denotes the relative closure of $\mathscr{R}$ as a subset of $\mathbb{S}^{2}$. If, however, $f(q)$ were contained in $\partial \mathscr{B}_{\vartheta}(\xi) \cap \mathbb{S}^{e}$, then we could conclude by (4.6) that $\gamma$ is tangent to $\partial \mathscr{B}_{\vartheta}(\xi)$ in $f(q)$, which implies that $q$ as the antipodal point of $f(q)$ is also contained in $\partial \mathscr{B}_{\vartheta}(\xi)$, a contradiction.

Therefore $f(q) \in \mathbb{S}^{w} \cap \overline{\mathscr{R}}$. Since $f(q) \in \gamma\left(\mathbb{S}^{1}\right)$ and since $\gamma\left(\mathbb{S}^{1}\right) \cap\left[\mathbb{S}^{w} \cap \overline{\mathscr{R}}\right]=$ $\tilde{\gamma}$ we have shown that $f$ is a continuous mapping from $\tilde{\gamma}$ to $\tilde{\gamma}$, and we may apply 
Brouwer's fixed point theorem to infer the existence of a point $q^{*} \in \tilde{\gamma}$ with $\eta_{q^{*}}(2 \vartheta)=f\left(q^{*}\right)=q^{*}=\eta_{q^{*}}(0)$, which would imply $2 \vartheta=2 \pi$ because $\eta_{q^{*}}$ parametrises a great circle on $\mathbb{S}^{2}$ with unit speed. But the last equation is absurd, since we assumed $\vartheta \leqq \pi / 2$.

Lemma 11. (Characteristic patterns of sphere filling curves II) Let $\gamma: \mathbb{S}^{1} \rightarrow \mathbb{S}^{2}$ be a closed rectifiable continuous curve with positive thickness $\Theta:=\Delta[\gamma]>0$, $\vartheta:=\arcsin \Theta \leqq \pi / 2$ such that $\mathscr{V}\left(T_{\vartheta}(\gamma)\right)=4 \pi$. Suppose that there is a point $\xi \in \mathbb{S}^{2}$ such that the intersection $\partial \mathscr{B}_{\vartheta}(\xi) \cap \gamma\left(\mathbb{S}^{1}\right)$ contains an open semicircle $S$, and let $\mathbb{S}^{w} \subset \mathbb{S}^{2}$ be the hemisphere containing $S$ such that $\partial \mathbb{S}^{w}$ intersects $\partial \mathscr{B}_{\vartheta}(\xi)$ orthogonally. Then there exists an $n \in \mathbb{N}$ such that $\vartheta=\pi /(2 n)$, and

$$
\gamma\left(\mathbb{S}^{1}\right) \cap \mathbb{S}^{w}=\bigcup_{i=1}^{n} \partial \mathscr{B}_{(2 i-1) \vartheta}(\xi) \cap \mathbb{S}^{w} .
$$

In other words, if $\gamma$ contains one semicircle $S=\mathbb{S}^{w} \cap \partial \mathscr{B}_{\vartheta}(\xi)$, then $\gamma\left(\mathbb{S}^{1}\right) \cap \mathbb{S}^{w}$ consists of a whole stack of latitudinal semicircles with mutual spherical distance $2 \vartheta$.

Proof. If $\vartheta=\pi / 2$ we find $n=1$ and $\gamma\left(\mathbb{S}^{1}\right)=\partial \mathscr{B}_{\vartheta}(\xi)$ is the only admissible curve, and (4.12) follows.

For $\vartheta \in(0, \pi / 2)$ there exists $n=n(\vartheta) \in \mathbb{N} \backslash\{1\}$ so that $\vartheta \in\left[\frac{\pi}{2 n}, \frac{\pi}{2(n-1)}\right)$. We will show that $\vartheta=\pi /(2 n)$ and that (4.12) holds. Notice first that the spherical torus property (ST) (see Remark 2.2) applied to any point $q \in S_{1}:=S$ implies

$$
\gamma\left(\mathbb{S}^{1}\right) \cap \mathscr{B}_{\vartheta}(\xi)=\emptyset .
$$

For an arbitrary point $p \in S_{1}$, consider the unit speed geodesic $\eta_{p}:[0,2 \pi] \rightarrow \mathbb{S}^{2}$ starting in $p$ in the direction orthogonal to $S_{1}$, that is, with $\eta_{p}(0)=p, \eta_{p}^{\prime}(0) \perp S_{1}$ at $p$ and $\left|\eta_{p}^{\prime}\right| \equiv 1$ on $[0,2 \pi]$, so that for all $s \in(0,2 \pi-2 \vartheta)$ the point $\eta_{p}(s)$ is contained in the open region $\mathbb{S}^{2} \backslash \overline{\mathscr{B}_{\vartheta}(\xi)}$. Hence $S_{1}$, and therefore $\gamma$, is tangent to the geodesic circle $\partial \mathscr{B}_{\vartheta}\left(\eta_{p}(\vartheta)\right)$ in the point $p$. This implies by means of the spherical torus property (ST)

$$
\mathscr{B}_{\vartheta}\left(\eta_{p}(\vartheta)\right) \cap \gamma\left(\mathbb{S}^{1}\right)=\emptyset \quad \text { for all } p \in S_{1} \text {. }
$$

According to Lemma 9 there is at least one point $\tilde{p} \in \partial \mathscr{B}_{\vartheta}\left(\eta_{p}(\vartheta)\right) \cap \gamma\left(\mathbb{S}^{1}\right) \backslash\{p\}$. If $\tilde{p}$ is not antipodal to $p$ on $\partial \mathscr{B}_{\vartheta}\left(\eta_{p}(\vartheta)\right)$, that is if $\tilde{p} \neq \eta_{p}(2 \vartheta)$, then $p$ and $\tilde{p}$ are contained in a closed semicircle by virtue of options (b) and (c) in Lemma 9. Therefore we find a point $q \in \partial \mathscr{B}_{\vartheta}\left(\eta_{p}(\vartheta)\right) \cap \gamma\left(\mathbb{S}^{1}\right) \backslash\{p\}$ sufficiently close to $p$ such that the unit speed geodesic $\tau_{q}:[0,2 \pi] \rightarrow \mathbb{S}^{2}$ with $\tau_{q}(0)=q, \tau_{q}^{\prime}(0) \perp \partial \mathscr{B}_{\vartheta}\left(\eta_{p}(\vartheta)\right)$ at $q, \tau_{q}(s) \in \mathbb{S}^{2} \backslash \overline{\mathscr{B}_{\vartheta}\left(\eta_{p}(\vartheta)\right)}$ for all $s \in(0,2 \pi-2 \vartheta)$, intersects $S_{1}$ sufficiently early, that is, such that

$$
\tau_{q}(\sigma) \in S_{1} \subset \gamma\left(\mathbb{S}^{1}\right) \text { for some } \sigma \in(0, \vartheta) .
$$

But this implies $\gamma\left(\mathbb{S}^{1}\right) \cap \mathscr{B}_{\vartheta}\left(\tau_{q}(\vartheta)\right) \neq \emptyset$ contradicting the spherical torus property (ST) at the point $q \in \gamma\left(\mathbb{S}^{1}\right)$. Hence we have shown that

$$
\partial \mathscr{B}_{\vartheta}\left(\eta_{p}(\vartheta)\right) \cap \gamma\left(\mathbb{S}^{1}\right)=\left\{p, \eta_{p}(2 \vartheta)\right\} \quad \text { for all } p \in S_{1} \text {. }
$$


Since $S_{1}$ is a semicircle contained in $\partial \mathscr{B}_{\vartheta}(\xi)$, the set

$$
S_{2}:=\bigcup_{p \in S_{1}} \eta_{p}(2 \vartheta) \subset \gamma\left(\mathbb{S}^{1}\right)
$$

is an open semicircle contained in $\partial_{\mathscr{B}} \mathscr{B}_{3}(\xi)$ unless $\vartheta=\pi / 3$ (hence $n=2$ ) in which case $S_{2}$ degenerates to a single point, namely the antipodal point $\bar{\xi}$ of $\xi$. But this is absurd taking (4.14) into account, since $\gamma$ is a closed curve with a continuous tangent.

We proceed with this construction by setting

$$
S_{i}:=\bigcup_{p \in S_{1}} \eta_{p}(2(i-1) \vartheta) \quad \text { for } i=3, \ldots, n \text {, }
$$

and we have $\eta_{p}^{\prime}(2(i-2) \vartheta) \perp S_{i-1}$ at the points $\eta_{p}(2(i-2) \vartheta)$ for $i=3, \ldots, n$, so that for all $s \in(2(i-2) \vartheta, 2 \pi-2(i-2) \vartheta)$ the point $\eta_{p}(s)$ is contained in $\mathbb{S}^{2} \backslash \overline{\mathscr{B}_{2(i-2) \vartheta}(\xi)}$. Hence $S_{i-1}$, and therefore $\gamma$, is tangent to each of the geodesic circles $\partial \mathscr{B}_{\vartheta}\left(\eta_{p}((2 i-3) \vartheta)\right)$ in the point $\eta_{p}(2(i-2) \vartheta)$ for $i=3, \ldots, n$, which implies by means of the spherical torus property (ST)

$$
\mathscr{B}_{\vartheta}\left(\eta_{p}((2 i-3) \vartheta)\right) \cap \gamma\left(\mathbb{S}^{1}\right)=\emptyset \quad \text { for all } p \in S_{1}, i=3, \ldots, n \text {. }
$$

Using Lemma 9, as before, we can prove for each $p \in S_{1}$ that

$\partial \mathscr{B}_{\vartheta}\left(\eta_{p}((2 i-3) \vartheta)\right) \cap \gamma\left(\mathbb{S}^{1}\right)=\left\{\eta_{p}(2(i-2) \vartheta), \eta_{p}((2 i-2) \vartheta)\right\}$ for $i=3, \ldots, n$.

Each of the sets $S_{i}$ is an open semicircle contained in $\partial \mathscr{B}_{(2 i-1) \vartheta}(\xi) \cap \mathbb{S}^{w}$, $i=1, \ldots, n-1$, since

$$
\vartheta+(2 i-2) \vartheta \leqq(2 n-3) \vartheta<\frac{2 n-3}{2 n-2} \pi<\pi \quad \text { for all } i=1, \ldots, n-1
$$

If $\vartheta=\pi /(2 n-1)$ then $S_{n}$ degenerates to a single point (since $\left.\vartheta+(2 n-2) \vartheta=\pi\right)$, which contradicts (4.15) for $i=n$, in combination with the fact that $\gamma$ is closed and has a continuous tangent.

If $\vartheta \in(\pi /(2 n-1), \pi /(2 n-2))$, then $\vartheta+(2 n-2) \vartheta=(2 n-1) \vartheta>\pi$, so that $S_{n} \subset \mathbb{S}^{e}$ is an open semicircle of spherical radius $(2 n-1) \vartheta-\pi<\vartheta$ about the antipodal point $\bar{\xi}$ of $\xi$, contradicting the definition of thickness $\Theta=\Delta[\gamma]=\sin \vartheta$.

If $\vartheta \in(\pi /(2 n), \pi /(2 n-1))$, then $\vartheta+(2 n-2) \vartheta<\pi$, hence $S_{n} \subset \mathbb{S}^{w}$ is an open semicircle of spherical radius $\pi-(2 n-1) \vartheta<\vartheta$ about $\bar{\xi}$, again contradicting the definition of thickness.

The only remaining angle is $\vartheta=\pi /(2 n)$, so that $S_{n} \subset \mathbb{S}^{w}$ is an open semicircle of spherical radius $\pi-(2 n-1) \vartheta=\pi /(2 n)$ about $\bar{\xi}$, and we have (4.12) in virtue of (4.15), if we add the endpoints of the open semicircles $S_{i}, i=1, \ldots, n$, using the continuity of $\gamma$.

Now we conclude this section with the 
Proof of Theorem 4. If $\vartheta=\pi / 2$ we find $n=1$, and the only possible solution is the equator $\beta^{1,0}$, and we are done.

For $\vartheta \in(0, \pi / 2)$ there is $k=k(\vartheta) \in \mathbb{N} \backslash\{1,2\}$ such that $2(k-1) \vartheta<2 \pi \leqq 2 k \vartheta$.

First we are going to prove that there is a closed semicircle $S$ contained in $\gamma\left(\mathbb{S}^{1}\right)$. For this purpose we fix $p \in \gamma\left(\mathbb{S}^{1}\right)$ and define $\eta_{p}:[0,2 \pi] \rightarrow \mathbb{S}^{2}$ to be the unit speed geodesic starting in $p$ in the direction orthogonal to $\gamma$, that is, with $\eta_{p}(0)=p, \eta_{p}^{\prime}(0) \perp \gamma$ at $p$ and $\left|\eta_{p}^{\prime}\right| \equiv 1$ on $[0,2 \pi]$. The spherical torus property (ST) applied to $p$ implies

$$
\gamma\left(\mathbb{S}^{1}\right) \cap \mathscr{B}_{\vartheta}\left(\eta_{p}(\vartheta)\right)=\emptyset .
$$

According to Lemma 9 either $\eta_{p}(2 \vartheta) \notin \gamma\left(\mathbb{S}^{1}\right)$, in which case $p$ is contained in a closed semicircle $S \subset \mathscr{B}_{\vartheta}\left(\eta_{p}(\vartheta)\right)$ with

$$
S \subset \gamma\left(\mathbb{S}^{1}\right)
$$

or $\eta_{p}(2 \vartheta) \in \gamma\left(\mathbb{S}^{1}\right)$. In the latter case, (4.16) implies that $\gamma$ is tangent to $\partial \mathscr{B}_{\vartheta}\left(\eta_{p}(\vartheta)\right)$, that is, $\eta_{p}^{\prime}(2 \vartheta) \perp \gamma$ at $\eta_{p}(2 \vartheta)$. In this way we investigate the whole collection of balls $\mathscr{B}_{\vartheta}\left(\eta_{p}((2 i-1) \vartheta)\right)$ for $i=1, \ldots, k$, and we claim that either we find a closed semicircle $S$ on one of the geodesic circles $\partial \mathscr{B}_{\vartheta}\left(\eta_{p}((2 i-1) \vartheta)\right)$, $i=1, \ldots, k$, or $\vartheta=\pi / k$, and

$$
\eta_{p}(2 i \vartheta) \in \gamma\left(\mathbb{S}^{1}\right), \quad \eta_{p}(2 k \vartheta)=p, \quad \eta_{p}^{\prime}(2 i \vartheta) \perp \gamma \text { at } \eta_{p}(2 i \vartheta)
$$

for $i=1, \ldots, k$. But (4.18) describes exactly the situation assumed in Lemma 10 so that we can conclude the existence of a closed semicircle $S$ of spherical radius $\vartheta$ in each of the two hemispheres bounded by $\eta_{p}([0,2 \pi])$.

To prove the claim we assume that none of the circles $\partial \mathscr{B}_{\vartheta}\left(\eta_{p}((2 i-1) \vartheta)\right)$, $i=1, \ldots, k$, contains a closed semicircle $S \subset \gamma\left(\mathbb{S}^{1}\right)$. Then we can apply Lemma 9 and the torus property successively - as demonstrated once above for $i=1-$ to conclude that

$$
\gamma\left(\mathbb{S}^{1}\right) \cap \bigcup_{i=1}^{k} \mathscr{B}_{\vartheta}\left(\eta_{p}((2 i-1) \vartheta)\right)=\emptyset .
$$

This implies that $2 \pi=2 k \vartheta$, since the inequality $(2 k-2) \vartheta<2 \pi<2 k \vartheta$ leads to a contradiction: If $(2 k-1) \vartheta \geqq 2 \pi$, then $(2 k-1) \vartheta-2 \pi<\vartheta$, which implies

$$
p \in \mathscr{B}_{\vartheta}\left(\eta_{p}((2 k-1) \vartheta)\right)
$$

contradicting (4.19). If $(2 k-1) \vartheta<2 \pi$, then $2 \pi-(2 k-1) \vartheta<\vartheta$, which leads to (4.20) as well, again contradicting (4.19). Hence we have shown $\vartheta=\pi / k$ and the properties (4.18) follows from our construction. As in the proof of Lemma 10 we find $k=2 n$ for some $n \in \mathbb{N} \backslash\{1\}$, since $\gamma$ is simple and closed.

Having established the existence of a closed semicircle $S$ contained in $\gamma\left(\mathbb{S}^{1}\right) \cap$ $\mathscr{B}_{\vartheta}\left(\eta_{p}((2 j-1) \vartheta)\right)$ for (at least) one $j \in\{1, \ldots, 2 n\}$, we can use Lemma 11 to conclude that

$$
\gamma\left(\mathbb{S}^{1}\right) \cap \mathbb{S}^{w}=\bigcup_{i=1}^{n} \partial \mathscr{B}_{(2 i-1) \vartheta}\left(\eta_{p}((2 j-1) \vartheta)\right) \cap \mathbb{S}^{w},
$$


where $\mathbb{S}^{w}$ denotes the hemisphere containing $S$ such that $\partial \mathbb{S}^{w}$ intersects $\partial \mathscr{B} \vartheta\left(\eta_{p}\right.$ $((2 j-1) \vartheta))$ orthogonally.

With that knowledge we observe that the intersection $\gamma\left(\mathbb{S}^{1}\right) \cap \partial \mathbb{S}^{w}$ consists of $2 n$ equidistant points in which $\gamma$ intersects the plane containing $\mathbb{S}^{w}$ orthogonally. Therefore Lemma 10 is applicable to conclude the existence of an open semicircle $S^{*}$ of spherical radius $\vartheta$ contained in $\gamma\left(\mathbb{S}^{1}\right) \cap \mathbb{S}^{e}$, where $\mathbb{S}^{e}:=\mathbb{S}^{2} \backslash \mathbb{S}^{w}$. Again by Lemma 11, one finds that also

$$
\gamma\left(\mathbb{S}^{1}\right) \cap \mathbb{S}^{e}=\bigcup_{i=1}^{n} \partial \mathscr{B}_{(2 i-1) \vartheta}(\xi) \cap \mathbb{S}^{e} \text { for some } \xi \in \mathbb{S}^{e} .
$$

But we have shown in Section 2 that the only possible closed and simple curves made of two stacks of equidistant latitudinal semicircles are the curves $\beta^{n, k}$.

\section{Open curves}

We now want to sketch that a similar construction is also possible for open curves. A tubular neighbourhood of an open curve $\gamma$ in $\mathbb{R}^{3}$ consists of three parts: a half-ball cap at the beginning and at the end of the curve and a middle part consisting of the disjoint union of normal discs of given radius centred at the curve.

Problem 1 (P'). Given a constant $\Omega \in(0,1]$, find a curve $\gamma_{\Omega}$ in the class

$\mathcal{C}_{\Omega}^{\prime}:=\left\{\gamma \in C^{1,1}\left(I, \mathbb{R}^{3}\right):|\gamma|=1 \&\left|\gamma^{\prime}\right|>0\right.$ on $\left.I, \triangle[\gamma] \geqq \Omega,|\gamma(1)-\gamma(0)| \geqq 2 \Omega\right\}$ with $I=[0,1]$ such that $\mathscr{L}\left(\gamma_{\Omega}\right)=\sup _{\mathcal{C}^{\prime} \Omega} \mathscr{L}$.

With only slight modifications using the additional assumption on the endpoints of the curves in competition, one can prove as in Theorem 1 the existence of solutions for Problem (P') for any given $\Omega \in(0,1]$. Here, the crucial $C^{1,1}$-estimate from [13, Lemma 2] for closed curves leading to (3.20) in the proof of Theorem 1 is replaced by the corresponding $C^{1,1}$-estimate proved in [8, Satz 2.14] for open curves.

A variant of Proposition 1 (Hotelling's theorem) for open curves implies that the volume of the middle part is, again, proportional to the length of the curve, while the volume of the caps stays fixed for fixed radius.

For certain values $\omega_{m}=\pi / m, 2 \leqq m \in \mathbb{N}$ of spherical thickness, one can perform a similar construction as in the case of closed curves. There are in fact two slightly different situations, depending on whether $m$ is even or odd.

(i) For $m$ even, consider $m / 2$ semicircles with spherical distance $2 \omega_{m}$ stacked up on the western hemisphere as described in the introduction. For the eastern hemisphere we take the north pole as a single point, together with $m / 2-1$ stacked up semicircles with spherical distance $2 \omega_{m}$ and, finally, the south pole as a second single point. After turning the eastern hemisphere by the angle $\omega_{m}$, all endpoints of the western semicircles match with the semicircle endpoints and the two single points on the eastern hemisphere. Turning further by the amount of $k \cdot 2 \omega_{m}$, $k \in\{0, \ldots, m / 2-1\}$, one can try to construct a single open connected curve $\alpha^{m, k}$. 
Note that the first member of this family (for $m=2, k=0$ ) is a semi great-circle, which is easily seen to be the unique solution for thickness $\omega_{2}=\pi / 2$.

(ii) For $m$ odd, start with a single point $C_{0}$ in the north pole, then stack up $(m-1) / 2$ circles $C_{i}$ of spherical radius $2 \omega_{m}$ around the north pole with the last circle $C_{m-1}$ around the south pole of spherical radius $\omega_{m}$. The discontinuous curve has a tubular neighbourhood of spherical thickness $\omega_{m}$ (note that the neighbourhood about the point $C_{0}$ is just a geodesic ball). Next, cut the sphere along a plane through the poles (cutting $C_{0}$ in two 'half-points') and turn by $k \cdot 2 \omega_{m}$. We denote this possibly discontinuous curve again by $\alpha^{m, k}$.

As in the proof of Lemma 1 one can show that the tubular neighbourhood remains a tubular neighbourhood of the same spherical radius after the turning process and the neighbourhoods of the 'half-points' form the spherical caps about the endpoints of the curve. To investigate under which circumstances the resulting curves $\alpha^{m, k}$ constitute one open connected arc, we use the same algebraic methods as in Section 2.

Lemma 12. For every even $m \in \mathbb{N}$ and $k \in\{0, \ldots, m / 2-1\}$ with $\operatorname{gcd}(2 k-$ $1, m)=1$, the appropriately re-parametrised curve $\alpha^{m, k}: I \rightarrow \mathbb{S}^{2}$ is a connected open, piecewise circular curve whose constant speed parametrisation is of class $C^{1,1}\left(I, \mathbb{R}^{3}\right)$ satisfying

$$
\triangle\left[\alpha^{m, k}\right]=\Omega_{m}=\sin \omega_{m}=\sin \frac{\pi}{m} .
$$

Moreover, for distinct $k_{1}, k_{2} \in\{0, \ldots, m / 2-1\}$ the curves $\alpha^{m, k_{1}}$ and $\alpha^{m, k_{2}}$ are not equivalent. There are $\varphi(m)$ distinct open connected curves for each $m$.

Proof. As mentioned above, only the algebraic arguments need to be adjusted to the present situation of open curves. Set $n:=m / 2 \in \mathbb{N}$. First consider the western hemisphere $\mathbb{S}^{w}$ and number the $2 n$ endpoints of the (all proper) semicircles counter-clockwise from 0 to $2 n-1$, such that checkpoint number $i$ and $2 n-i-1$ correspond to the $i$ th semicircle $(i=0, \ldots, n-1)$; see Fig. 6 . For the eastern hemisphere, $\mathbb{S}^{e}$, number the points from 0 to $2 n-1$, where 0 and $n$ correspond to the single points, while $i$ and $2 n-i$ correspond to the endpoints of the $i$ th semi-circle $(i=1, \ldots, n-1)$. When turning the hemisphere $\mathbb{S}^{e}$ by multiples of $2 \omega_{2 n}$, the curve closes again in a nice $C^{1}$-fashion (note that $\mathbb{S}^{e}$ was already turned once by $\omega_{2 n}$ to align the endpoints during construction). Since there are only two endpoints, we will arrive at one open curve and, possibly, several closed curves.

The semicircles on $\mathbb{S}^{w}$ connect the checkpoints to $n$ pairs, which is a permutation on the checkpoints $w(i) \equiv-i-1 \bmod 2 n$, so if we pass through checkpoint $i$ along the curve we will next pass through checkpoint $w(i)$. Similarly, the eastern hemisphere defines $e(i) \equiv-i \bmod 2 n$. The twist by $k \cdot 2 \omega_{n}$ is again described by $t^{k}(i) \equiv i+k \bmod 2 n$. As we pass along the curve $\alpha^{m, k}$ we run alternately through the semicircles on each hemisphere. If we just entered a hemisphere through checkpoint $i$, we will enter it the next time at the checkpoint $t^{-k} \circ e \circ t^{k} \circ w(i)$. For $q$ we find the formula

$$
q(i) \equiv t^{-k} \circ e \circ t^{k} \circ w(i) \equiv-((-i-1)+k)-k \equiv i-(2 k-1) \bmod 2 n
$$


and after l-steps $q^{l}(i) \equiv i-(2 k-1) l \bmod 2 n$. In order to see whether $\alpha^{m, k}$ is one connected open curve, we start at checkpoint 0 (which is one end) and note through which checkpoints we pass. The run has to be reflected at the other endpoint, so that we pass through the curve in two directions, passing each checkpoint on the component, not only even or odd ones as in Lemma 1. So $\alpha^{m, k}$ is one connected open curve if and only if $q$ consists of one cycle of length $2 n$. By Lemma 2 this is the case if and only if $\operatorname{gcd}(2 k-1,2 n)=1$.

To count the solutions, note that $\operatorname{gcd}(2 k, 2 n) \geqq 2$ and therefore

$$
\begin{aligned}
& \#\{k \in\{1, \ldots, n\}: \operatorname{gcd}(2 k-1,2 n)=1\} \\
& \quad=\#\{k \in\{1, \ldots, 2 n-1\}: \operatorname{gcd}(k, 2 n)=1\}=\varphi(m) .
\end{aligned}
$$

Each of the $\alpha^{m, k}$ is easily seen to be unique up to rigid motions.

Lemma 13. For every odd $m \in \mathbb{N}$ and $k \in\{0, \ldots, m-1\}$ with $\operatorname{gcd}(2 k, m)=1$ the appropriately re-parametrised curve $\alpha^{m, k}: I \rightarrow \mathbb{S}^{2}$ is a connected open, piecewise circular curve whose constant speed parametrisation is of class $C^{1,1}\left(I, \mathbb{R}^{3}\right)$ satisfying

$$
\triangle\left[\alpha^{n, k}\right]=\Omega_{m}=\sin \omega_{m}=\sin \frac{\pi}{m} .
$$

Moreover, for distinct $k_{1}, k_{2} \in\{0, \ldots, m-1\}$ the curves $\alpha^{m, k_{1}}$ and $\alpha^{m, k_{2}}$ are not equivalent. There are $\varphi(m)$ distinct open connected curves for each $m$.

Proof. Set $n:=(m-1) / 2 \in \mathbb{N}$. As in the proof of Lemma 12, we check the order in which the curve passes certain checkpoints and whether we can reach every checkpoint in one run.

First consider the western hemisphere $\mathbb{S}^{w}$ and number the $2 n+1$ endpoints of the semicircles counter-clockwise from 0 to $2 n$, such that the checkpoint numbers $i$ and $2 n+1-i$ correspond to the $i$ th semicircle $(i=1, \ldots, n-1)$ and $i=0$ corresponds to the single point. When turning the hemisphere $\mathbb{S}^{e}$ by multiples of $2 \omega_{n}$, the curve closes again in a nice $C^{1}$-fashion. Since there are only two endpoints, we will end up with at most one open connected curve and, possibly, several closed curves. In the extreme case $k=0$, the open curve degenerates to a point and we have $n$ closed circles.

The semicircles and the single point on the western hemisphere act again as a permutation $c(i) \equiv-i \bmod 2 n+1$ on the checkpoints. The turn by $k \cdot 2 \omega_{n}$ is again described by $t^{k}(i) \equiv i+k \bmod 2 n+1$. As we pass along the curve $\alpha^{m, k}$ we run alternately through the semicircles on each hemisphere. If we just entered a hemisphere through checkpoint $i$, we will enter it the next time at the checkpoint $t^{-k} \circ c \circ t^{k} \circ c(i)$. For $q$ we find the formula

$$
q(i) \equiv t^{-k} \circ c \circ t^{k} \circ c(i) \equiv-((-i)+k)-k \equiv i-2 k \quad \bmod 2 n+1
$$

and after 1-steps $q^{l}(i) \equiv i-2 k l \bmod 2 n+1$. In order to see whether $\alpha^{m, k}$ is one connected open curve, we start at checkpoint 0 (which is one endpoint) and note through which checkpoints we pass. The run has to be reflected at the other 
endpoint, so that we pass through the curve in two directions, passing each checkpoint on the component, not only even or odd ones as in Lemma 1. So $\alpha^{m, k}$ is one connected open curve if and only if $q$ consists of one cycle of length $m=2 n+1$. By Lemma 2 this is the case if and only if $\operatorname{gcd}(2 k, 2 n+1)=\operatorname{gcd}(2 k, m)=1$. To count the number of solutions, note that $\operatorname{gcd}(2 k, 2 n+1)=\operatorname{gcd}(k, 2 n+1)$, since $2 n+1$ is odd, and therefore

$$
\begin{aligned}
& \#\{k \in\{1, \ldots, m-1\}: \operatorname{gcd}(2 k, m)=1\} \\
& =\#\{k \in\{1, \ldots, m-1\}: \operatorname{gcd}(k, m)=1\}=\varphi(m) .
\end{aligned}
$$

Again, we can use the fact that the tubular neighbourhood of $\alpha^{m, k}$ covers the whole sphere to prove that they are the unique solutions:

Lemma 14. (Characterisation of open sphere filling curves) For an open rectifiable continuous curve $\gamma: I \rightarrow \mathbb{S}^{2}$ with positive thickness $\Omega:=\Delta[\gamma] \in(0,1)$, $\omega:=\arcsin \Omega \in(0, \pi / 2)$, and with $|\gamma(0)-\gamma(1)| \geqq 2 \Omega$ the following two statements are equivalent:

(i) $\mathscr{V}\left(T_{\omega}(\gamma)\right)=4 \pi$;

(ii) For any $\xi \in \mathbb{S}^{2}$ such that $\mathscr{B}_{\omega}(\xi) \cap \gamma(I)=\emptyset$ one of the following is true:

(a) $\partial \mathscr{B}_{\omega}(\xi) \cap \gamma(I)=\{P, Q\}$ with $\operatorname{dist}_{\mathbb{S}^{2}}(P, Q)=2 \omega$ (antipodal points); $\gamma$ is tangential to $\partial \mathscr{B}_{\omega}(\xi)$ in at least one of the points $P$ or $Q$;

(b) $\partial \mathscr{B}_{\omega}(\xi) \cap \gamma\left(\mathbb{S}^{1}\right)=\{$ semicircle of spherical radius $\omega\}$.

Proof. The situation for open curves $\gamma$ differs from that in Proposition 2 only in the possibility that, apart from $\gamma$ connecting two points along $\partial \mathscr{B}_{\omega}(\xi)$, these two points could both be two endpoints of great arcs on $\gamma$ (see [8, Satz 3.27]). However, this case cannot happen here since we have $|\gamma(1)-\gamma(0)| \geqq 2 \Omega$.

The cases (ii) $\Rightarrow$ (i) and (i) $\Rightarrow$ (b) and the first part of (a) are proved as in Lemma 9. If $P$ or $Q$ is an inner point of $\gamma$ the tangency is evident. Assume that $P$ and $Q$ are the endpoints of $\gamma$, both not tangential. Then we could consider the great circle $\eta:(-\pi, \pi) \rightarrow \mathbb{S}^{2}, \eta(0)=\rho$ intersecting the shorter great arc from $P$ to $Q$ orthogonally in $\rho$. Then $\mathscr{B}_{\omega+\epsilon}(\eta(\epsilon))$ would not intersect $\gamma$ for small $\epsilon>0$ which would contradict Part (i).

Theorem 6. (Sphere filling open thick curves) If $\mathscr{V}\left(T_{\omega}(\gamma)\right)=4 \pi$ for $\omega \in(0, \pi / 2]$ and some open curve $\gamma \in \mathcal{C}^{\prime} \Omega$ with $\Omega=\sin \omega \in(0,1]$, then there is some $m \in \mathbb{N}$ and $k \in\{0, \ldots, m / 2\}$ with $\operatorname{gcd}(2 k-1, m)=1$ if $m$ is even, or $k \in\{0, \ldots, m-1\}$ with $\operatorname{gcd}(2 k, m)=1$ if $m$ is odd, such that (i) $\omega=\omega_{m}$, (ii) $\triangle[\gamma]=\Omega_{m}$, where $\Omega_{m}=\sin \omega_{m}$, and (iii) $\gamma=\alpha^{m, k}$.

Proof. We will only sketch the proof, since the proof for open curves is slightly easier than for closed curves. Indeed, we have two natural points to start our construction if we equip $\gamma$ with an orientation, namely the start- and endpoints of the curve. Let $\eta_{p, t}$ be the unit speed great-circle going through $\eta_{p, t}(0)=p \in \mathbb{S}^{2}$ with $\eta_{p, t}^{\prime}=t \in \mathbb{S}^{2}$. By Lemma 14 we have $\eta_{\gamma(0), t}(2 \omega) \in \gamma(I)$ for $t \cdot \gamma^{\prime}(0) \leqq 0$. If 
$\omega=\pi / 2$ then $\eta_{\gamma(0), t}(2 \omega)=\gamma(1)$ is the other endpoint, and $\gamma=\alpha^{1,0}$ (otherwise we would have $|\gamma(0)-\gamma(1)|<\Omega$ contradicting the fact that $\gamma \in \mathcal{C}_{\Omega}^{\prime}$ ). If $\omega<\pi / 2$ the set $\left\{\eta_{\gamma(0), t}(2 \omega): t \cdot \gamma^{\prime}(0) \leqq 0, t \in \mathbb{S}^{2}\right\}$ will be a semicircle. As in Lemma 11 , we can continue this process stacking up semi-circles until we arrive either at

(i) a semi-circle of spherical radius $\omega$, which implies $\omega=\pi / m$ for some odd $m \in \mathbb{N}$.

(ii) or a single point, which has to be $\gamma(1)$. This implies $\omega=\pi / m$ for some even $m \in \mathbb{N}$.

In case (i) we can redo the construction with the endpoint and find the two hemispheres each filled with $(m-1) / 2$ stacked up semi-circles and one single point. Therefore $\gamma$ must be equivalent to $\alpha^{m, k}$ for some odd $m$ and some $k \in \mathbb{N}$. In case (ii) both end caps are contained in one hemisphere, and in the boundary of this hemisphere we find the characteristic pattern, so we can apply Lemma 10 and then 11 to see that the other hemisphere must consist of stacked up semi-circles, so $\gamma$ is again equivalent to $\alpha^{m, k}$ for an even $m$ and some $k \in \mathbb{N}$. In both cases (i) and (ii) the additional restrictions on $k$ depending on whether $m$ is even or odd are derived in Lemmas 12 and 13.

Acknowledgments. The first author would like to thank Professor Micheal Farber and Dr. Torsten Schöneborn, whose comments led to Proposition 2 and a simplification of the uniqueness proof, respectively. The second author would like to thank the EPFL Lausanne, in particular Professor John H. Maddocks, and also the Centro di Ricerca Matematica Ennio de Giorgi at the Scuola Normale Superiore in Pisa, specifically Professor Mariano Giaquinta, for their hospitality. In addition, we thank the DFG (project no. Mo 966/4-1) and the SNF (project no. 107878 and 117898) for generously supporting this research.

\section{References}

1. Bach, E., Shallit, J.: Algorithmic Number Theory. MIT Press Cambridge, Massachusetts, 1996

2. Cantarella, J., Fu, J.H.G., Kusner, R.B., Sullivan, J.M., Wrinkle, N.C.: Criticality for the Gehring link problem. Geom. Topol. 10, 2055-2116 (2006)

3. Cantarella, J., Kusner, R.B., Sullivan, J.M.: On the minimum ropelength of knots and links. Invest. Math. 150, 257-286 (2002)

4. Cantarella, J., Piatek, M., Rawdon, E.: Visualizing the tightening of knots. ViS'05: Proceedings of the 16th IEEE Visualization 2005, 575-582. IEEE Computer Society, Washington, DC, 2005

5. Carlen, M., Laurie, B., Maddocks, J.H., Smutny, J.: Biarcs, global radius of curvature, and the computation of ideal knot shapes. Physical and Numerical Models in Knot Theory, Ser. on Knots and Everything 36 (Eds. Calvo M. and Rawdon S.) World Scientific, Singapore, 75-108, 2005

6. Do Carmo, M.P.: Differential Geometry of Curves and Surfaces. Prentice Hall, New Jersey, 1976

7. Durumeric, O.C.: Local structure of ideal shapes of knots. Top. Appl. 154, 3070-3089 (2007)

8. GeRlach, H.: Der Globale Krümmungsradius für offene und geschlossene Kurven im $\mathbb{R}^{N}$. Diploma thesis at Bonn University, 2004. http://www.littleimpact.de/permanent/ math/2009/dipl/ 
9. Gerlach, H.: Ideal Knots and other packing problems of tubes. $\mathrm{PhD}$ thesis No. 4601, EPFL Lausanne (2010). http://library.epfl.ch/theses/?display=detail\&nr=4601

10. Gerlach, H.: Construction of sphere-filling ropes. Website: http://www.littleimpact. de/permanent/math/sphere_filling/

11. Gonzalez, O., De la Llave, R.: Existence of ideal knots. J. Knot Theory Ramif. 12, $123-133$ (2003)

12. Gonzalez, O., Maddocks, J.H.: Global curvature, thickness and the ideal shapes of knots. Proc. Natl. Acad. Sci. USA 96, 4769-4773 (1999)

13. Gonzalez, O., Maddocks, J.H., Schuricht, F., von der Mosel, H.: Global curvature and self-contact of nonlinearly elastic curves and rods. Calc. Var. 14, 29-68 (2002)

14. Gray, A.: Tubes., 2nd edn. Progress in Mathematics, 221. Birkhäuser Verlag, Basel, 2004

15. Hotelling, H.: Tubes and spheres in n-spaces. Am. J. Math. 61, 440-460 (1939)

16. Katzav, E., Adda-Bedia, M., Boudaoud, A.: A statistical approach to close packing of elastic rods and to DNA packaging in viral capsids. Proc. Natl. Acad. Sci. USA 103, 18900-18904 (2006)

17. Kusner, R.B.: On thickness and packing density for knots and links. Physical Knots: Knotting, Linking, and Folding Geometric Objects in $\mathbb{R}^{3}$ (Las Vegas, NV, 2001) (Eds. Calvo M. and Rawdon S.) AMS Providence, Contemp. Math. 304, 175-180, (2002)

18. Pireranski, P.: In search of ideal knots. Ideal Knots, Ser. on Knots and Everything 19 (Eds. Stasiak, Katritch and Kauffman) World Scientific, Singapore, 20-41, 1998

19. Smutny, J.: Global radii of curvature and the biarc approximation of spaces curves: in pursuit of ideal knot shapes. PhD thesis No. 2981, EPFL Lausanne (2004). http:// library.epfl.ch/theses/?display=detail\&nr $=2981$

20. Schuricht, F., von der Mosel, H.: Global curvature for rectifiable loops. Math. Z. 243, 37-77 (2003)

21. Schuricht, F., von der Mosel, H.: Characterization of ideal knots. Calc. Var. Partial Differ. Equ. 19, 281-305 (2004)

22. STRZelecki, P., von Der Mosel, H.: On rectifiable curves with $L^{p}$-bounds on global curvature: self-avoidance, regularity, and minimizing knots. Math. Z. 257, 107-130 (2007)

23. Varea, C., Aragon, J.L., Barrio, R.A.: Turing patterns on a sphere. Phys. Rev. E 60, 4588-4592 (1999)

24. WeYl, H.: On the volume of tubes. Am. J. Math. 61, 461-472 (1939)

25. Wiggs, C.C., TAYlor, C.J.C.: Bead puzzle. US Patent D269629 (issued 1983)

EPFL, SB IMB LCVMM, Bâtiment MA,

Station 8, 1015 Lausanne, Switzerland. e-mail: henryk.gerlach@epfl.ch

and

Institut für Mathematik, RWTH Aachen University,

Templergraben 55, 52062 Aachen, Germany.

e-mail: heiko@instmath.rwth-aachen.de

(Received April 24, 2009 / Accepted November 3, 2010)

Published online January 8, 2011 - (C) Springer-Verlag (2011) 